\title{
Ground State Entropy of the Potts Antiferromagnet on Triangular Lattice Strips
}

\author{
Shu-Chiuan Changf and Robert Shrock网 \\ C. N. Yang Institute for Theoretical Physics \\ State University of New York \\ Stony Brook, N. Y. 11794-3840
}

\begin{abstract}
We present exact calculations of the zero-temperature partition function (chromatic polynomial) $P$ for the $q$-state Potts antiferromagnet on triangular lattice strips of arbitrarily great length $L_{x}$ vertices and of width $L_{y}=3$ vertices and, in the $L_{x} \rightarrow \infty$ limit, the exponent of the ground-state entropy, $W=e^{S_{0} / k_{B}}$. The strips considered, with their boundary conditions $(B C)$ are (a) $\left(F B C_{y}, P B C_{x}\right)=$ cyclic, (b) $\left(F B C_{y}, T P B C_{x}\right)=$ Möbius, (c) $\left(P B C_{y}, P B C_{x}\right)=$ toroidal, and (d) $\left(P B C_{y}, T P B C_{x}\right)=$ Klein bottle, where $F, P$, and $T P$ denote free, periodic, and twisted periodic. Exact calculations of $P$ and $W$ are also given for wider strips, including (e) cyclic, $L_{y}=4$, and (f) $\left(P B C_{y}, F B C_{x}\right)=$ cylindrical, $L_{y}=5$, 6. Several interesting features are found, including the presence of terms in $P$ proportional to $\cos \left(2 \pi L_{x} / 3\right)$ for case (c). The continuous locus of points $\mathcal{B}$ where $W$ is nonanalytic in the $q$ plane is discussed for each case and a comparative discussion is given of the respective loci $\mathcal{B}$ for families with different boundary conditions. Numerical values of $W$ are given for infinite-length strips of various widths and are shown to approach values for the $2 \mathrm{D}$ lattice rapidly. A remark is also made concerning a zero-free region for chromatic zeros.
\end{abstract}

*email: shu-chiuan.chang@sunysb.edu

**email: robert.shrock@sunysb.edu 


\section{Introduction}

The $q$-state Potts antiferromagnet (AF) [1, 2] exhibits nonzero ground state entropy, $S_{0}>0$ (without frustration) for sufficiently large $q$ on a given lattice $\Lambda$ or, more generally, on a graph $G$. This is equivalent to a ground state degeneracy per site $W>1$, since $S_{0}=$ $k_{B} \ln W$. Such nonzero ground state entropy is important as an exception to the third law of thermodynamics. There is a close connection with graph theory here, since the zerotemperature partition function of the above-mentioned $q$-state Potts antiferromagnet on a graph $G$ satisfies

$$
Z(G, q, T=0)_{P A F}=P(G, q)
$$

where $P(G, q)$ is the chromatic polynomial expressing the number of ways of coloring the vertices of the graph $G$ with $q$ colors such that no two adjacent vertices have the same color (for reviews, see [3]-[6]). The minimum number of colors necessary for such a coloring of $G$ is called the chromatic number, $\chi(G)$. Thus'

$$
W(\{G\}, q)=\lim _{n \rightarrow \infty} P(G, q)^{1 / n}
$$

where $n=v(G)$ is the number of vertices of $G$ and $\{G\}=\lim _{n \rightarrow \infty} G$. Since $P(G, q)$ is a polynomial, one can generalize $q$ from $\mathbb{Z}_{+}$to $\mathbb{C}$. The zeros of $P(G, q)$ in the complex $q$ plane are called chromatic zeros; a subset of these may form an accumulation set in the $n \rightarrow \infty$ limit, denoted $\mathcal{B}$, which is the continuous locus of points where $W(\{G\}, q)$ is nonanalytic. I The maximal region in the complex $q$ plane to which one can analytically continue the function $W(\{G\}, q)$ from physical values where there is nonzero ground state entropy is denoted $R_{1}$. The maximal value of $q$ where $\mathcal{B}$ intersects the (positive) real axis is labelled $q_{c}(\{G\})$. This point is important since it separates the interval $q>q_{c}(\{G\})$ on the positive real $q$ axis where the Potts model (with $q$ extended from $\mathbb{Z}_{+}$to $\mathbb{R}$ ) exhibits nonzero ground state entropy (which increases with $q$, asymptotically approaching $S_{0}=k_{B} \ln q$ for large $q$, and which for a regular lattice $\Lambda$ can be calculated approximately via large $-q$ series expansions) from the interval $0 \leq q \leq q_{c}(\{G\})$ in which $S_{0}$ has a different analytic form.

\footnotetext{
${ }^{1}$ At certain special points $q_{s}$ (typically $q_{s}=0,1, . ., \chi(G)$ ), one has the noncommutativity of limits $\lim _{q \rightarrow q_{s}} \lim _{n \rightarrow \infty} P(G, q)^{1 / n} \neq \lim _{n \rightarrow \infty} \lim _{q \rightarrow q_{s}} P(G, q)^{1 / n}$, and hence it is necessary to specify the order of the limits in the definition of $W\left(\{G\}, q_{s}\right)[\overline{7}$. We use the first order of limits here; this has the advantage of removing certain isolated discontinuities in $W$.

${ }^{2}$ Although it does not happen in the cases considered here, for some families of graphs $\mathcal{B}$ may be null, and $W$ may also be nonanalytic at certain discrete points.
} 


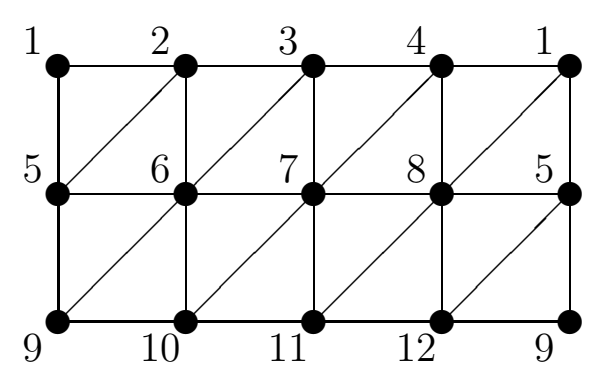

(a)

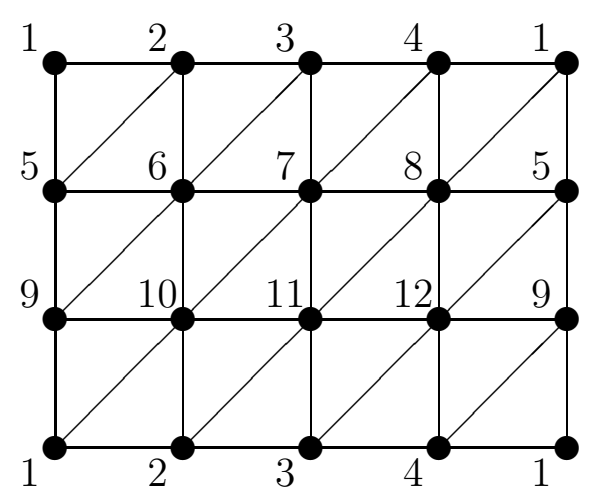

(c)

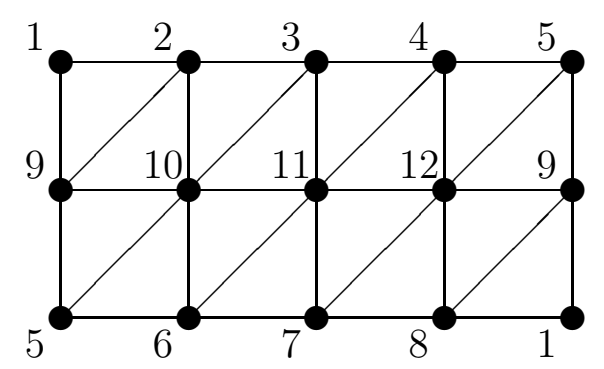

(b)

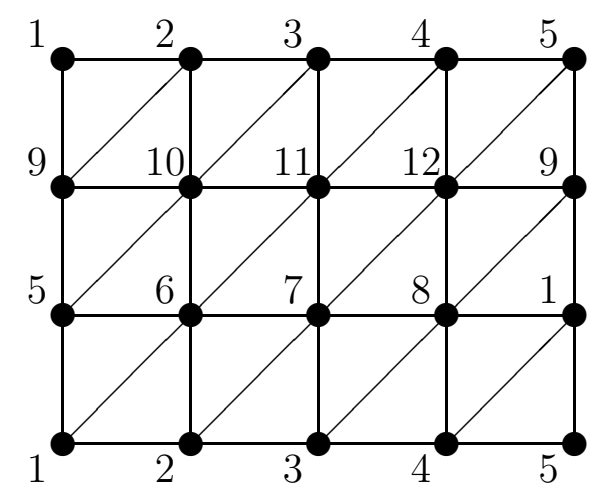

(d)

Figure 1: Illustrative strip graphs of the triangular lattice with width $L_{y}=3$ and length $L_{x}=4$ having the following boundary conditions: (a) $\left(F B C_{y}, P B C_{x}\right)=$ cyclic, (b) $\left(F B C_{y}, T P B C_{x}\right)=$ Möbius, (c) $\left(P B C_{y}, P B C_{x}\right)=$ toroidal, and $(\mathrm{d})\left(P B C_{y}, T P B C_{x}\right)=$ Klein bottle.

In the present work we report exact calculations of $P(G, q), W(\{G\}, q)$, and $\mathcal{B}$ for families of triangular lattice strips of fixed width $L_{y}=3$ vertices and arbitrary length $L_{x}=m$ vertices. The longitudinal and transverse directions are taken to be $\hat{x}$ and $\hat{y}$ ). In Fig. 1 we display some illustrative examples. A generic form for chromatic polynomials for recursively defined families of graphs, of which strip graphs $G_{s}$ are special cases, is

$$
P\left(\left(G_{s}\right)_{m}, q\right)=\sum_{j=1}^{N_{\lambda}} c_{j}(q)\left(\lambda_{j}(q)\right)^{m}
$$

where $c_{j}(q)$ and the $N_{\lambda}$ terms $\lambda_{j}(q)$ depend on the type of strip graph $G_{s}$ but are independent of $m$. 
Let us comment on the motivations for the current study and how it extends previous work. Calculations of the chromatic polynomials for the cyclic and Möbius strip graphs of the square lattice were carried out for $L_{y}=2$ in [8] (see also [9, 10]). Subsequently, these calculations were extended to the width $L_{y}=3$ for the cyclic [11] and Möbius [12 square lattice strips. After studies of chromatic zeros in [13, 14] and, for $L_{y}=2$ strips in [8, 15, 16], the $W$ and $\mathcal{B}$ defined in the infinite-length limit were determined for $L_{y}=2$ in [7] and for $L_{y}=3$ in [11, 12]. Similar calculations were carried out for strips of various lattices with widths up to $L_{y}=4$ and free boundary conditions in [17, 18, 19, 20], and for cyclic and Möbius graphs involving homeomorphic expansions of square strips [21] and cyclic polygon chains 22]. An important question concerns the effect of boundary conditions (BC's), and hence graph topology, on $P, W$, and $\mathcal{B}$. We use the symbols $\mathrm{FBC}_{y}$ and $\mathrm{PBC}_{y}$ for free and periodic transverse boundary conditions and $\mathrm{FBC}_{x}, \mathrm{PBC}_{x}$, and $\mathrm{TPBC}_{x}$ for free, periodic, and twisted periodic longitudinal boundary conditions. The term "twisted" means that the longitudinal ends of the strip are identified with reversed orientation. These strip graphs can be embedded on surfaces with the following topologies: (i) $\left(\mathrm{FBC}_{y}, \mathrm{FBC}_{x}\right)$ : open strip; (ii) $\left(\mathrm{PBC}_{y}, \mathrm{FBC}_{x}\right)$ : cylindrical; (iii) $\left(\mathrm{FBC}_{y}, \mathrm{PBC}_{x}\right)$ : cylindrical, denoted cyclic here; (iv) $\left(\mathrm{FBC}_{y}, \mathrm{TPBC}_{x}\right)$ : Möbius; (v) $\left(\mathrm{PBC}_{y}, \mathrm{PBC}_{x}\right)$ : torus; and (vi) $\left(\mathrm{PBC}_{y}, \mathrm{TPBC}_{x}\right)$ : Klein bottle.3 (We recall that that unlike graphs of type (i)-(v), the Klein bottle surface cannot be embedded without self-intersection in $\mathbb{R}^{3}$.)

Several specific effects of different boundary conditions have been found (for a general discussion, see [23, 25]). First, from comparisons of exact calculations of $W$ for the infinitelength limit of lattice strips with boundary conditions of types (i)-(iv), it was observed that those with a global circuitf lead to a locus $\mathcal{B}$ that separates the $q$ plane into different regions, and it was inferred that the presence of global circuits is a sufficient condition for $\mathcal{B}$ to have this property [17, 19, 21, 22, 11, 12. . For strip graphs of regular lattices, this is equivalent to having $P B C_{x}$ or $T P B C_{x}$, i.e., periodic or twisted periodic boundary conditions in the direction in which the strip length goes to infinity as $L_{x} \rightarrow \infty$. Further support for this inference was adduced from the calculation of $P, W$, and $\mathcal{B}$ for the $L_{y}=3$ strip of the square lattice with torus and Klein bottle boundary conditions [26]. Applications of a coloring matrix method [10] for these calculations has been discussed in [27, 28]. For the (homogeneous) strip graphs with $\left(\mathrm{FBC}_{y}, \mathrm{FBC}_{x}\right)$ in [17, 19], $\mathcal{B}$ consists of arcs that do not

\footnotetext{
${ }^{3}$ These BC's can all be implemented in a manner that is uniform in the length $L_{x}$; the case (vii) $\left(\mathrm{TPBC}_{y}, \mathrm{TPBC}_{x}\right)$ with the topology of the projective plane requires different identifications as $L_{x}$ varies and will not be considered here.

${ }^{4} \mathrm{~A}$ global circuit is a route following a lattice direction which has the topology of $S^{1}$ and a length $\ell_{g . c}$. that goes to infinity as $n \rightarrow \infty$.

${ }^{5}$ This is not a necessary condition, as was shown in [18].
} 
separate regions of the $q$ plane. As the width of the strip increases, these arcs tend to elongate, and their ends tend to move toward each other, thereby suggesting that if one considered the sequence of strip graphs of this type with width $L_{y}$ (having taken the limit $L_{x} \rightarrow \infty$ to obtain a locus $\mathcal{B}$ for each member of this sequence), then in the limit $L_{y} \rightarrow \infty$, the arcs would close to form a $\mathcal{B}$ that separated the $q$ plane into two or more regions and passed through $q=0$. The interesting feature of the families of graphs with global circuits is that when the graphs contain a global circuit, this separation of the $q$ plane into regions (with $\mathcal{B}$ passing through $q=0$ ) already occurs for finite $L_{y}$. This means that the $W$ functions of the infinite-length limit of these graphs with cyclic and twisted cyclic longitudinal boundary conditions already exhibit a feature which is expected to occur for the $\mathcal{B}$ for the $W$ function of the full two-dimensional lattice. This expectation is supported by the calculation of $W$ and $\mathcal{B}$ for the 2D triangular lattice with cylindrical boundary conditions by Baxter [29]. Thus, although calculations of chromatic polynomials for lattice strips with global circuits are, in general, more difficult than for the corresponding strips with free boundary conditions, such calculations are worthwhile, since in the $L_{x} \rightarrow \infty$ limit, the resultant locus $\mathcal{B}$ embodies the analytic property of $\mathcal{B}$ expected for the full $2 \mathrm{D}$ lattice, viz., that it crosses the real axis at least at $q=0$ and a maximal point $q_{c}$, and separates the $q$ plane into different regions.

Second, it has been found that for a given type of strip graph $G_{s}$ with $\mathrm{FBC}_{y}$, the chromatic polynomials for $\mathrm{PBC}_{x}$ and $\mathrm{TPBC}_{x}$ boundary conditions (i.e., cyclic and Möbius strips) have the same $\lambda_{j}$, although in general different $c_{j}$. It follows that the loci $\mathcal{B}$ are the same for these two different longitudinal boundary condition choices [21, 11, 12. In the case of $\mathrm{PBC}_{y}$, the reversal of orientation involved in going from $\mathrm{PBC}_{x}$ to $\mathrm{TPBC}_{x}$ longitudinal boundary conditions (i.e. from torus to Klein bottle topology) can lead to the removal of some of the $\lambda_{j}$ 's that were present; i.e., $P$ for the $\left(\mathrm{PBC}_{y}, \mathrm{TPBC}_{x}\right)$ strip may involve only a subset of the $\lambda_{j}$ 's that are present for the $\left(\mathrm{PBC}_{y}, \mathrm{PBC}_{x}\right)$ strip. For example, for the $L_{y}=3$ strips of the square lattice with $\left(\mathrm{PBC}_{y}, \mathrm{PBC}_{x}\right)$ boundary conditions, there are $N_{\lambda}=8 \lambda_{j}$ 's, but for the strip with $\left(\mathrm{PBC}_{y}, \mathrm{TPBC}_{x}\right)$ boundary conditions only a subset of $N_{\lambda}=5$ of these terms occurs in $P$ [26]. None of the three $\lambda_{j}$ 's that are absent from $P$ in the $\mathrm{TPBC}_{x}$ case is leading, so that $\mathcal{B}$ is the same for both of these families.

Third, for a given type of strip graph $G_{s}$ containing a global circuit, it has been found that, in the infinite-length limit where the locus $\mathcal{B}$ is defined, it not only passes through the origin, $q=0$, but always crosses the positive real axis at one or more points, the maximal one being denoted $q_{c}(\{G\})$, as mentioned above. In contrast, for strip graphs that do not contain global circuits, $\mathcal{B}$ may not cross the real axis. For example, for the (infinite-length limit of the) strip of the triangular lattice with free transverse and longitudinal boundary conditions, $\left(F B C_{y}, F B C_{x}\right)$, the locus $\mathcal{B}$ crosses the real axis for width $L_{y}=3$ but does not 
cross it for $L_{y}=4$ (see Fig. 5 of [17]). For square strips with $\left(F B C_{y},(T) P B C_{x}\right)$ boundary conditions, it has been found that $q_{c}(\{G\})$ is a nondecreasing function of $L_{y}$ for the cases studied so far, namely $q_{c}=2$ for $L_{y}=1$ and $L_{y}=2$, and $q_{c} \simeq 2.33654$ for $L_{y}=3$ [11, 12, 23]. For the strips of the triangular lattice with $\left(F B C_{y},(T) P B C_{x}\right)$ boundary conditions, we only have one width for which $\mathcal{B}$ has been determined, namely, $L_{y}=2$, for which $q_{c}=3$. It is thus of interest to explore what the value of $q_{c}$ is for cyclic and Möbius strips of the triangular lattice with larger widths. For the $L_{y}=3$ torus and Klein bottle strips of the square lattice, very interestingly, $q_{c}=3$, which is the same value as for the $2 \mathrm{D}$ square lattice 26 . Clearly it is worthwhile to investigate what the value of $q_{c}$ is for torus and Klein bottle strips of the triangular lattice, and we shall do this here.

Fourth, for the strip graph $\left(G_{s}\right)_{m}$ with a given type of transverse boundary conditions $\mathrm{BC}_{y}$, the chromatic polynomial for $\mathrm{PBC}_{x}$ has a larger value of $N_{\lambda}$ than the chromatic polynomial for $\mathrm{FBC}_{x}$, and the corresponding loci $\mathcal{B}$ are different.

Fifth, one may ask how the $W$ functions in region $R_{1}$ compare for different boundary conditions. It has been found [21, 22, 11, 12, 23] that, for a given type of strip graph $G_{s}$, in the region $R_{1}$ (with its left-hand boundary on the positive real $q$ axis at $q_{c}$ ) being defined for the $\mathrm{PBC}_{x}$ boundary conditions, the $W$ function is the same for $\mathrm{FBC}_{x}, \mathrm{PBC}_{x}$, and $\mathrm{TPBC}_{x}$. This includes the region of real $q$ greater than this value of $q_{c}$, so that this result is somewhat reminiscent of the statement of the existence of the thermodynamic limit in statistical mechanics, i.e. the fact that in the disordered phase of a statistical mechanical system the thermodynamic functions are independent of the boundary conditions and if an ordered phase exists, then the role of the boundary conditions is only to set a preferred direction for the symmetry-breaking order parameter.

Our new results on $P, W$, and $\mathcal{B}$ for the $L_{y}=3$ strip of the triangular lattice with the various boundary conditions of type (iii)-(vi) give insight concerning these five items, as do our additional results for $L_{y}=4$ cyclic strips and $L_{y}=5,6$ cylindrical strips. Calculations of these quantities for the $L_{y}=2$ cyclic and Möbius triangular strips were presented in [11]. An early study of $P$ for the $L_{y}=2$ strip is in [30] and a coloring matrix method to obtain $P$ has been given in 27]. $P, W$, and $\mathcal{B}$ have been calculated for triangular lattice strips with free boundary conditions (i) for $L_{y}$ up to 4 [17]. (Some related calculations including work on cases with noncompact $\mathcal{B}$, are [16, 7], [31]-34].)

For strips with global circuits, and $L_{x}$ above the lowest values involving degenerate cases, the triangular lattice strips with boundary conditions of type (i)-(vi) have $n=L_{y} L_{x}$ vertices;

\footnotetext{
${ }^{6}$ We note the following corrections in Sand's result for $P$ for the $L_{y}=2$ cyclic strip of the triangular lattice, given on p. 88 of [30]: the coefficients of the last two terms should be reversed in sign to read $(z \equiv q$ in his notation $)(z-1)\left[[(1 / 2)(5-2 z+\sqrt{9-4 z})]^{m}+[(1 / 2)(5-2 z-\sqrt{9-4 z})]^{m}\right]$, as in eq. (5.23) of [11].
} 
the cyclic and Möbius triangular strips have $e=\left(3 L_{y}-2\right) L_{x}$ edges while the torus and Klein bottle triangular strips have $e=3 L_{y} L_{x}$ edges. Define $N_{t}$ to be the number of triangles in the strips (i.e., excluding the triangles forming the cross sections of the strips in the case $\left.L_{y}=3\right)$; then, again for $L_{x}$ and $L_{y}$ large enough to avoid degenerate cases, the cyclic and Möbius strips have $N_{t}=2\left(L_{y}-1\right) L_{x}$ such triangles, while the torus and Klein bottle strips have $2 L_{y} L_{x}$ such triangles. For strips of type (i), $e=3 L_{x} L_{y}-2 L_{x}-2 L_{y}+1$ and $N_{t}=2\left(L_{y}-1\right)\left(L_{x}-1\right)$ while for strips of type (ii), $e=3 L_{x} L_{y}-2 L_{y}$ and $N_{t}=2 L_{y}\left(L_{x}-1\right)$.

Again, for $L_{x}=m$ and $L_{y}$ large enough to avoid degenerate cases, the chromatic number for the triangular lattice strips with cyclic or torus boundary conditions is

$$
\chi\left(\operatorname{tri}\left(L_{y}\right)_{m}, F B C_{y}, P B C_{x}\right)=\chi\left(\operatorname{tri}\left(L_{y}\right)_{m}, P B C_{y}, P B C_{x}\right)= \begin{cases}3 & \text { if } m=0 \text { mod } 3 \\ 4 & \text { if } m=1 \text { or } 2 \bmod 3\end{cases}
$$

and for the triangular lattice strips with Möbius or Klein bottle boundary conditions,

$$
\chi\left(\operatorname{tri}\left(L_{y}\right)_{m}, F B C_{y}, T P B C_{x}\right)=\chi\left(\operatorname{tri}\left(L_{y}\right)_{m}, P B C_{y}, T P B C_{x}\right)=4 \quad \forall \quad m \geq 3 .
$$

For strips with $\left(F B C_{y}, F B C_{x}\right)$ or $\left(P B C_{y}, F B C_{x}\right), \chi=3$.

\section{$2 \quad L_{y}=3$ Cyclic and Möbius Triangular Strips}

We calculate the chromatic polynomials by iterated use of the deletion-contraction theorem, via a generating function approach [17, 19]. In general, for a strip graph of type $G_{s}$ we have

$$
\Gamma\left(G_{s}, q, x\right)=\sum_{m=m_{0}}^{\infty} P\left(\left(G_{s}\right)_{m}, q\right) x^{m-m_{0}}
$$

where, as before [11], we take $m_{0}=2$ for strips with periodic or twisted periodic longitudinal boundary conditions. The generating functions $\Gamma\left(G_{s}, q, x\right)$ are rational functions of the form

$$
\Gamma\left(G_{s}, q, x\right)=\frac{\mathcal{N}\left(G_{s}, q, x\right)}{\mathcal{D}\left(G_{s}, q, x\right)}
$$

with

$$
\mathcal{N}\left(G_{s}, q, x\right)=\sum_{j=0}^{d_{\mathcal{N}}} A_{G_{s}, j}(q) x^{j}
$$

and

$$
\mathcal{D}\left(G_{s}, q, x\right)=1+\sum_{j=1}^{d_{\mathcal{D}}} b_{G_{s}, j}(q) x^{j}
$$


where the $A_{G_{s}, i}$ and $b_{G_{s}, i}$ are polynomials in $q$ (with no common factors) and

$$
d_{\mathcal{N}}=\operatorname{deg}_{x}(\mathcal{N})
$$

and

$$
d_{\mathcal{D}}=\operatorname{deg}_{x}(\mathcal{D}) .
$$

The denominator $\mathcal{D}$ can be written in factorized form as

$$
\mathcal{D}\left(G_{s}, q, x\right)=\prod_{j=1}^{d_{\mathcal{D}}}\left(1-\lambda_{G_{s}, j} x\right) .
$$

Equivalently, the $\lambda_{G_{s}, j}$ are roots of the equation

$$
\xi^{d_{\mathcal{D}}} \mathcal{D}\left(G_{s}, q, 1 / \xi\right)=\xi^{d_{\mathcal{D}}}+\sum_{j=1}^{d_{\mathcal{D}}} b_{G_{s}, j} \xi^{d_{\mathcal{D}}-j} .
$$

From the generating function, we calculate the chromatic polynomials in the form of eq. (1.3) by the use of the general formula, eq. (2.14), in [19],

$$
P\left(G_{m}, q\right)=\sum_{j=1}^{d_{\mathcal{D}}}\left[\sum_{s=0}^{d_{\mathcal{N}}} A_{s} \lambda_{j}^{d_{\mathcal{D}}-s-1}\right]\left[\prod_{1 \leq i \leq d_{\mathcal{D}} ; i \neq j} \frac{1}{\left(\lambda_{j}-\lambda_{i}\right)}\right] \lambda_{j}^{m-m_{0}}
$$

(where the conventions in eq. (2.1) were chosen such that $m_{0}=0$ in [19]). Note that some of the coefficients $c_{G_{s}, j}$ may vanish, so that not all of the $\lambda_{G_{s}, j}$ 's in $\mathcal{D}\left(G_{s}, q, x\right)$ contribute to the sum in (1.3) [11. The formula (1.3) for the chromatic polynomial has the virtue of being a closed-form expression. However, for a given type of strip graph, for values of the width $L_{y}$ greater than the first one or two values, the set of $\lambda_{j}$ 's include nonpolynomial algebraic roots. These occur, of course, as symmetric functions of the roots, so that, by a theorem on symmetric functions of roots of algebraic equations, their resultant contributions to $P$ are expressible in terms of the coefficients of these equations (which are polynomials in $q$ ) and hence are polynomials in $q$ [12]. However, in calculating chromatic polynomials and the resultant chromatic zeros for large values of $L_{x}=m$, it can be convenient to use directly the expression for the generating function, since both the numerator and denominator of this function, eqs. (2.3), (2.4), are polynomials in $q$ so that one does not have to rely upon cancellations of nonpolynomial algebraic expressions at intermediate stages in the calculation.

The coefficients $c_{t r i\left(L_{y}\right), j}$ that enter into the expressions for the chromatic polynomial (1.3) for the cyclic triangular $(t)$ strip of width $L_{y}$ are (like those for the cyclic square strip) 
certain polynomials that we denote $c^{(d)}$, given by 35

$$
c^{(d)}=\prod_{k=1}^{d}\left(q-q_{d, k}\right)
$$

where

$$
q_{d, k} \equiv 2+2 \cos \left(\frac{2 \pi k}{2 d+1}\right) \quad \text { for } \quad k=1,2, \ldots d
$$

with $0 \leq d \leq L_{y}$. We list below the specific $c^{(d)}$ 's that appear in our results for the $L_{y}=3$ and $L_{y}=4$ cyclic strips of the triangular lattice

$$
\begin{gathered}
c^{(0)}=1, \quad c^{(1)}=q-1, \quad c^{(2)}=q^{2}-3 q+1, \\
c^{(3)}=q^{3}-5 q^{2}+6 q-1,
\end{gathered}
$$

and

$$
c^{(4)}=(q-1)\left(q^{3}-6 q^{2}+9 q-1\right) .
$$

Where the coefficient $c^{(0)}$ appears in chromatic polynomials, we shall simply write it as unity.

For the cyclic $L_{y}=3$ triangular strip of length $L_{x}=m$, we obtain the result

$$
\begin{aligned}
& P\left(\operatorname{tri}(3 \times m), F B C_{y}, P B C_{x}, q\right)=\sum_{j=1}^{10} c_{t 3, j}\left(\lambda_{t 3, j}\right)^{m} \\
= & c^{(3)}(-1)^{m}+c^{(2)}\left[(q-2)^{m}+\left(\lambda_{t 3,3}\right)^{m}+\left(\lambda_{t 3,4}\right)^{m}\right] \\
+ & c^{(1)}\left[[-(q-2)(q-3)]^{m}+\left(\lambda_{t 3,6}\right)^{m}+\left(\lambda_{t 3,7}\right)^{m}+\left(\lambda_{t 3,8}\right)^{m}\right]+\left[\left(\lambda_{t 3,9}\right)^{m}+\left(\lambda_{t 3,10}\right)^{m}\right] .
\end{aligned}
$$

where the short notation $t 3$ is used in subscripts for $\operatorname{tr} i\left(L_{y}=3\right)$. The labelling of $c_{t 3, j}$ and $\lambda_{t 3, j}$ in eq. (2.15) is consecutive, so that $c_{t 3,1}=c^{(3)}, \lambda_{t 3,1}=-1 ; c_{t 3,2}=c^{(2)}, \lambda_{t 3,2}=q-2$, and so forth:

$$
\lambda_{t 3,(3,4)}=\frac{1}{2}(-7+2 q \pm \sqrt{25-8 q}),
$$

$\lambda_{t 3, j}, j=6,7,8$ are the roots of the cubic equation

$$
\xi^{3}+b_{t 3,1} \xi^{2}+b_{t 3,2} \xi+b_{t 3,3}=0
$$

where

$$
b_{t 3,1}=2 q^{2}-12 q+19
$$




$$
\begin{gathered}
b_{t 3,2}=(q-2)\left(q^{3}-9 q^{2}+28 q-29\right) \\
b_{t 3,3}=-(q-2)^{4}(q-3),
\end{gathered}
$$

and

$$
\lambda_{t 3,(9,10)}=\frac{1}{2}\left[q^{3}-7 q^{2}+18 q-17 \pm\left(q^{6}-14 q^{5}+81 q^{4}-250 q^{3}+442 q^{2}-436 q+193\right)^{1 / 2}\right] .
$$

Note that some $\lambda_{t 3, j}$ 's vanish if $q=2$ or $q=3$; specifically, if $q=2$, then $\lambda_{t 3,3}=0$ and two of the roots of the cubic equation (2.17) vanish, while for $q=3, \lambda_{t 3,3}=0$ and one of the roots of eq. (2.17) vanish. The vanishing of the chromatic polynomial at $q=0,1,2$ and (i) $q=3$ for the cyclic strip with $m \neq 0 \bmod 3$ or (ii) $q=3$ for the Möbius strip discussed below involves both some terms $\lambda_{j}$ vanishing and cancellations among others. This is also true of chromatic polynomials for strips of other lattices.

Parenthetically, we note that the denominator of the generating function for this strip graph actually has $d_{\mathcal{D}}=16$ :

$$
\mathcal{D}\left(\operatorname{tri}\left(L_{y}=3\right), F B C_{y}, P B C_{x}, q, x\right)=\sum_{j=1}^{16}\left(1-\lambda_{t 3, j}(q) x\right)
$$

where the $\lambda_{t, j}$ were given in eq. (2.15) and

$$
\begin{gathered}
\lambda_{t, 11}=\lambda_{t, 1}=-1 \\
\lambda_{t, 12}=\lambda_{t, 13}=q-3
\end{gathered}
$$

and $\lambda_{t, j}, j=14,15,16$ are the roots of the cubic equation

$$
\xi^{3}+(2 q-5)(q-3) \xi^{2}+(q-2)^{2}(q-3)(q-4) \xi-(q-2)^{4}(q-3)=0 .
$$

A new feature of these results that we have not encountered before in our calculations of chromatic polynomials and generating functions for strip graphs is the occurrence of factors in $\mathcal{D}$ that have multiplicity higher than unity: specifically, the factors $(1+x)^{2}$, corresponding to $\lambda_{t 3,1}=\lambda_{t 3,11}=-1$, and $[1-(q-3) x]^{2}$, corresponding to $\lambda_{t 3,12}=\lambda_{t 3,13}=q-3$. None of the $\lambda_{t 3, j}, 12 \leq j \leq 16$, contribute to $P$ (and $\lambda_{t 3,11}$ is identical to $\lambda_{t 3,1}$ ), so that $P$ only involves the ten $\lambda_{t 3, j}$ 's in eq. (2.15). We had found cases earlier of some $\lambda_{j}$ 's in $\mathcal{D}$ not contributing to $P$, e.g., for the $L_{y}=2$ cyclic strip of the kagomé lattice and homeomorphic expansions of the $L_{y}=2$ cyclic strip of the square lattice [11. However, this is the first value of $L_{y}$ where we have encountered this type of behavior for a strip of a homopolygonal lattice.]

\footnotetext{
${ }^{7}$ As in [36, 37], we define a homopolygonal 2D lattice as a regular tiling of the plane involving a single type of polygon, in which all vertices are equivalent, while a heteropolygonal lattice is the same but involving two or more different types of regular polygons. In the case of the kagomé lattice, these are triangles and hexagons.
} 
The numerator of the generating function has degree $\operatorname{deg}_{x}(\mathcal{N})=14$. The polynomials $A_{j}, j=1, . ., 14$ comprising this numerator can be calculated from the expressions for $P$ and $\mathcal{D}$, eqs. (2.15) and (2.22) and hence are not listed here.

From eq. (1.4), it follows that for $m=1$ or $2 \bmod 3$ and $q=3$, the chromatic polynomial in eq. (2.15) vanishes; for $m=0 \bmod 3$ (and $m \geq 3$ ) and $q=3$ we find

$$
P\left(\operatorname{tri}\left(L_{y}=3\right)_{m}, F B C_{y}, P B C_{x}, q=3\right)=6 \quad \text { for } \quad m=0 \quad \bmod 3 .
$$

This may be contrasted with the case of a $k$-critical graph $G_{k}$, where $\chi(G)=k$ and $P(G, q=$ $\chi(G))=k$ ! (e.g. $k=3$ for the triangular lattice).

We define

$$
C(G)=\sum_{j=1}^{N_{\lambda_{G}}} c_{G, j}
$$

where the $G$-dependence in the coefficients is indicated explicitly. Note that for recursive graphs like the strip graphs considered here, the $c_{G, j}$ depend on $L_{y}$ and the boundary conditions, but not on $L_{x}$. Our results above give

$$
C=q(q-1)^{2} \quad \text { for } \quad G=\operatorname{tri}\left(L_{y}=3, F B C_{y}, P B C_{x}\right)
$$

in accord with the generalization [11, 12] $C=P\left(T_{L_{y}}, q\right)$ for this strip, where $P\left(T_{n}, q\right)=$ $q(q-1)^{n-1}$ and $T_{n}$ is the tree graph with $n$ vertices. This was also true for the $L_{y}=3$ cyclic strip of the square lattice [11, 12] and is in accord with the coloring matrix approach [26, 27].

For the $L_{y}=3$ triangular lattice Möbius strip (denoted by the subscript $t 3 M b$ ) we calculate

$$
P\left(\operatorname{tri}\left(L_{y}=3\right)_{m}, F B C_{y}, T P B C_{x}, q\right)=\sum_{j=1}^{10} c_{t 3 M b, j}\left(\lambda_{t 3 M b, j}\right)^{m}
$$

where

$$
\lambda_{t 3 M b, j}=\lambda_{t 3, j}, \quad j=1, \ldots, 10 .
$$

As was true in the case of the $\mathrm{L}_{y}=2$ Möbius strip of the triangular lattice, the coefficients $c_{j}$ are not just of the $c^{(d)}$ polynomial form; instead, some coefficients are rational functions of $q$ and others are algebraic nonpolynomial functions of $q$ :

$$
\begin{gathered}
c_{t 3 M b, 1}=c^{(2)} \\
c_{t 3 M b, 2}=-\frac{\left(q^{2}-4 q+5\right)}{2(q-2)}
\end{gathered}
$$




$$
\begin{gathered}
c_{t 3 M b,(3,4)}=\frac{1}{4(q-2)}\left[(q-3)^{2} \mp \frac{(q-1)(q-5)}{\lambda_{t, 3}-\lambda_{t, 4}}\right] \\
c_{t 3 M b, 5}=\frac{(q-1)\left(q^{2}-4 q+5\right)}{2(q-2)} \\
c_{t 3 M b, 9}=c_{t 3 M b, 10}=c^{(0)}=1 .
\end{gathered}
$$

The $j=3,4$ terms can be written in a manner which manifestly exhibits their property of being a symmetric function of $\lambda_{t 3,3}$ and $\lambda_{t 3,4}$ :

$c_{t 3 M b, 3}\left(\lambda_{t 3,3}\right)^{m}+c_{t 3 M b, 4}\left(\lambda_{t 3,4}\right)^{m}=\frac{(q-3)^{2}}{4(q-2)}\left[\left(\lambda_{t 3,3}\right)^{m}+\left(\lambda_{t 3,4}\right)^{m}\right]-\frac{(q-1)(q-5)}{4(q-2)}\left[\frac{\left(\lambda_{t 3,3}\right)^{m}-\left(\lambda_{t 3,4}\right)^{m}}{\lambda_{t 3,3}-\lambda_{t 3,4}}\right]$.

It is convenient to leave the expressions for $c_{t 3 M b, j}, j=6,7,8$, in the general forms that follow from eq. (2.9):

$$
c_{t 3 M b, j}=\lambda_{t 3, j}^{-2}\left[\sum_{s=0}^{8} A_{t m 3, s}\left(\lambda_{t 3, j}\right)^{9-s}\right]\left[\prod_{1 \leq i \leq 10 ; i \neq j} \frac{1}{\left(\lambda_{t 3, j}-\lambda_{t 3, i}\right)}\right], \quad j=6,7,8
$$

where the $A_{t 3 M b, s}$ are given in the appendix.

For the sum of the coefficients for the $L_{y}=3$ Möbius strip of the triangular lattice we calculate

$$
C=\sum_{j=1}^{10} c_{t 3 M b, j}=q(q-1) \quad \text { for } \quad G=\operatorname{tri}\left(L_{y}=3, F B C_{y}, T P B C_{x}\right)
$$

in agreement with a general formula that we have derived elsewhere for a Möbius strip graph $G_{s}$ of width $L_{y}$ of the square or triangular lattice [35],

$$
C\left(G_{s}\left(L_{y}\right), M b\right)=\left\{\begin{array}{ll}
0 & \text { for even } L_{y} \\
P\left(T_{\left(\frac{L_{y}+1}{2}\right)}, q\right) & \text { for odd } L_{y}
\end{array} .\right.
$$

Chromatic zeros for the $L_{y}=3, L_{x}=m=20$ cyclic graph of the triangular lattice are shown in Fig. 2; with this value of $m$, the chromatic zeros for the triangular lattice Möbius graph are quite similar and both sets of chromatic zeros lie close to the boundary $\mathcal{B}$ and indicate its position. The locus $\mathcal{B}$ and the $W$ functions are the same for the cyclic and Möbius graph families. We find

$$
q_{c}\left(\operatorname{tri}\left(L_{y}=3\right), F B C_{y},(T) P B C_{x}\right)=3 .
$$




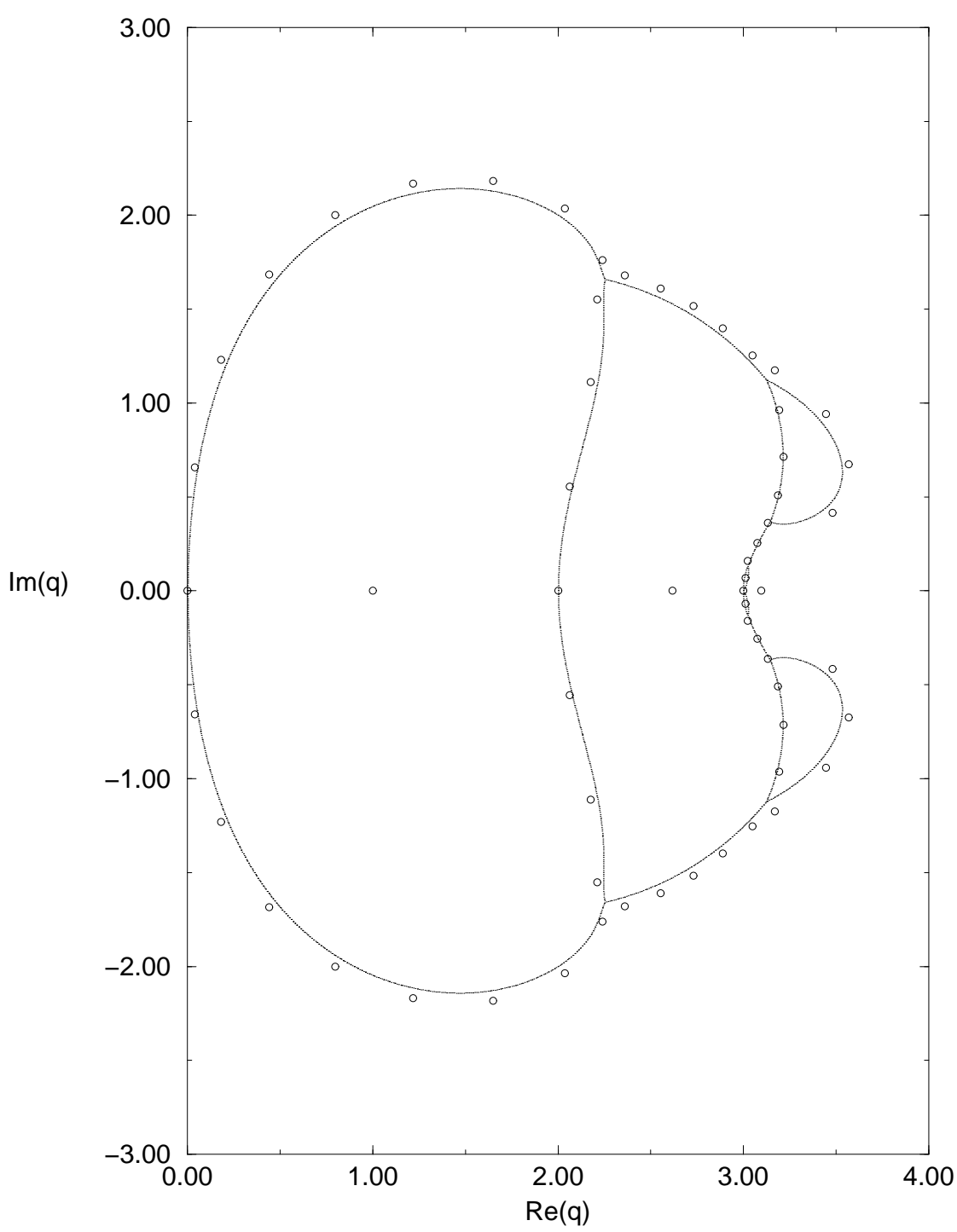

Figure 2: $\mathcal{B}$ and chromatic zeros for the $L_{y}=3, L_{x}=m=20$ (i.e., $n=60$ ) cyclic triangular strip graph. 
This will be discussed further below when we give our result for $q_{c}$ for the corresponding $L_{y}=4$ strip of the triangular lattice.

The locus $\mathcal{B}$ has support for $\operatorname{Re}(q) \geq 0$ and separates the $q$ plane into five main regions. The outermost one, region $R_{1}$, extends to infinite $|q|$ and includes the intervals $q \geq 3$ and $q \leq 0$ on the real $q$ axis. The other regions are labelled consequtively moving leftward along the real axis from $q=q_{c}=3$ to $q=0$, and then including complex conjugate pairs of regions that have no overlap with the real axis. Thus, region $R_{2}$ includes the real interval $2 \leq q \leq 3$, while region $R_{3}$ includes the real interval $0 \leq q \leq 2$. There are also two complex-conjugate regions, $R_{4}, R_{4}^{*}$ centered at approximately $q \simeq 3.35 \pm 0.7 i$. The triple points occur at (i) $q \simeq 3.14+0.37 i$ and (ii) $q \simeq 3.12+1.12 i$, where $R_{1}, R_{2}$, and $R_{4}$ are contiguous, and (iii) $q \simeq 2.25+1.66 i$, where $R_{1}, R_{2}$, and $R_{3}$ are contiguous, together with the complex conjugates of these points. In addition, there are two tiny regions $R_{5}, R_{5}^{*}$ that touch the real axis at the single point $q_{c}=3$ and extend a very short distance to the upper and lower right, with triple points at about $q \simeq 3.02 \pm 0.13 i$. These are plotted in Fig. 2. Thus, $q_{c}$ is a multiple point where four branches of $\mathcal{B}$ intersect. The occurrence of extremely small regions was also found for the $\left(L_{x} \rightarrow \infty\right.$ limit of the) $L_{y}=3$ cyclic strip of the square lattice, as shown in Fig. 1 of [11. The fact that such tiny regions can occur means that when one performs the usual computer scan over the complex plane to map out the region diagram and determine the dominant $\lambda_{j}$ 's in each region, since this involves a finite grid, one can only detect tiny sliver regions down to a certain resolution set by the grid of the scan. On the real axis, this is not a serious complication, since the mapping is a one-variable problem, but in the complex plane, one must a commensurately fine scanning grid to detect tiny sliver regions.

The part of $\mathcal{B}$ and the associated chromatic zeros with largest magnitude occur at roughly $q \simeq 3.53+0.69 i$ and have $|q| \simeq 3.59$. Comparing the present locus $\mathcal{B}$ for $L_{y}=3$ with the one obtained in [11] for $L_{y}=2$, we obtain further evidence supporting the conjecture that for a strip graph of a regular lattice with periodic or twisted periodic longitudinal boundary conditions ( $\mathcal{B}$ is the same for both of these), the envelope curve of $\mathcal{B}$, i.e. the outermost portion of $\mathcal{B}$ for a given $L_{y}$ surrounds the envelope curve for the $\mathcal{B}$ of the same strip with a smaller value of $L_{y}$. This was found to be true for the cyclic and Möbius strips of the square lattice for the cases $L_{y}=2,3$ and 4 (and, for the cyclic case, also $L_{y}=1$ ) [7, 11, 23]. For strips of a regular lattice $\Lambda$, as $L_{y} \rightarrow \infty$, one expects that the envelope curves defined for each $L_{y}$ will approach a limiting curve, which is precisely the envelope for the boundary $\mathcal{B}$ of the full $2 \mathrm{D}$ lattice $\Lambda$ defined via this limit.

In region $R_{1}, \lambda_{t 3,9}$ is the dominant $\lambda_{j}$ (with appropriate choice of branch cut for evaluation 
away from the negative real axis), so

$$
W=\left(\lambda_{t 3,9}\right)^{1 / 3}, \quad q \in R_{1} .
$$

The fact that this is the same as $W$ for the $\left(\mathrm{FBC}_{y}, \mathrm{FBC}_{x}\right)$ case [17] is a general result [23]. In region $R_{2}, \lambda_{t 3,4}$ is dominant, so

$$
|W|=\left|\lambda_{t 3,4}\right|^{1 / 3}, \quad q \in R_{2}
$$

(in regions other than $R_{1}$, only $|W|$ can be determined unambiguously [7]). In region $R_{3}, W$ is given by the largest (in magnitude) of the roots of the cubic (2.17), which we label $\lambda_{t 3,6}$ :

$$
|W|=\left|\lambda_{t 3,6}\right|^{1 / 3}, \quad q \in R_{3}
$$

In regions $R_{4}, R_{4}^{*}$,

$$
|W|=\left|\lambda_{t 3,8}\right|^{1 / 3}, \quad q \in R_{4}, R_{4}^{*} .
$$

where $\lambda_{t 3,8}$ is the other among these cubic roots that has maximal magnitude in this region. In $R_{5}, R_{5}^{*},|W|=\left|\lambda_{t 3,6}\right|^{1 / 3}$.

\section{$3 \quad L_{y}=4$ Cyclic Triangular Strip}

We have succeeded in performing an exact calculation of the chromatic polynomial for the cyclic triangular strip of arbitrarily great length and of the next larger width, namely, $L_{y}=4$. The calculation is considerably more involved than for the $L_{y}=3$ cyclic strip, as is indicated by the number of $\lambda_{j}$ terms in eq. (1.3), namely, $N_{\lambda}=26$, as contrasted with the value $L_{y}=10$ for the $L_{y}=3$ cyclic strip. Elsewhere we have proved that $N_{\lambda}$ is the same for the square and triangular strips of a given width and have given a general determination of $N_{\lambda}$ as a function of $L_{y}$ [35]. To go beyond this width for this family of cyclic strips of the triangular lattice would be increasingly difficult, since the number of terms $N_{\lambda}$ in (1.3) is 70, 192, and 534 for $L_{y}=5,6$, and 7 , respectively. For $L_{y}=4$ we find the chromatic polynomial

$$
P\left(\operatorname{tri}(4 \times m), F B C_{y}, P B C_{x}, q\right)=\sum_{j=1}^{26} c_{t 4, j}\left(\lambda_{t 4, j}\right)^{m}
$$

where one term is $\lambda_{t 4,1}=1$ and the others, $\lambda_{t 4, j}$, are the roots of (i) the quartic equation

(9.2.1), for $2 \leq j \leq 5$; (ii) the quartic equation (9.2.2), for $6 \leq j \leq 9$; (iii) the degree-8 equation (9.2.7), for $10 \leq j \leq 17$; and (iv) the degree-9 equation (9.2.16), for $18 \leq j \leq 26$. Since these equations are somewhat lengthy, they are given in the appendix. In contrast to 
the $L_{y}=2$ and $L_{y}=3$ cyclic triangular strips, here it is not possible to solve for all of the terms $\lambda_{t 4, j}$ as algebraic roots since for $j=10$ through $j=26$ these are roots of equations of degree higher than quartic. The corresponding coefficients $c_{t 4, j}$ are

$$
\begin{gathered}
c_{t 4,1}=c^{(4)} \\
c_{t 4, j}=c^{(0)} \quad \text { for } \quad 2 \leq j \leq 5 \\
c_{t 4, j}=c^{(3)} \quad \text { for } \quad 6 \leq j \leq 9 \\
c_{t 4, j}=c^{(2)} \quad \text { for } \quad 10 \leq j \leq 17 \\
c_{t 4, j}=c^{(1)} \quad \text { for } \quad 18 \leq j \leq 26 .
\end{gathered}
$$

As before, one can, equivalently, list the generating function; however, since all of the relevant information is present in (3.1) with the requisite definitions of the coefficients $c_{t 4, j}$ and terms $\lambda_{t 4, j}$, we shall forego this.

For the Möbius $L_{y}=4$ strip of the triangular lattice, a general argument [12] shows that the $\lambda_{j}$ 's are the same as for the cyclic strip, although the coefficients $c_{j}$ are different and are more complicated than those for the cyclic strip, as was already shown by the $L_{y}=2$ triangular strip, where two coefficients for the Möbius case are algebraic nonpolynomial functions of $q$. As discussed before, since $\mathcal{B}$ is determined only by the $\lambda_{j}$ 's, it is the same for the cyclic and Möbius strips of a given lattice.

Chromatic zeros for the $L_{y}=4, L_{x}=m=16$ (hence $n=64$ ) cyclic graph of the triangular lattice are shown in Fig. B; the complex zeros give an approximate indication of $\mathcal{B}$. The locus $\mathcal{B}$ separates the $q$ plane into six main regions, including four that contain intervals of the real axis. The outermost one, region $R_{1}$, extends to infinite $|q|$ and includes the intervals $q \geq q_{c}$ and $q \leq 0$ on the real $q$ axis. Region $R_{2}$ includes the real interval $3 \leq q \leq q_{c}$; region $R_{3}$ includes the real interval $2 \leq q \leq 3$, and region $R_{4}$ includes the real interval $0 \leq q \leq 2$. There are also complex-conjugate regions $R_{5}$ and $R_{5}^{*}$ centered at approximately $q \simeq 3.5 \pm 0.6 i$. There could also be other such complex-conjugate pairs of regions. The density of zeros is observed to be smaller on the parts of $\mathcal{B}$ extending through $q=2$ and $q=3$ and also on the right-most bubble-like curves than on the rest of the of $\mathcal{B}$. As before with the $L_{y}=3$ strip, it is straightforward to determine the various triple points. The maximal point at which the locus $\mathcal{B}$ crosses the real axis is

$$
q_{c}\left(\operatorname{tri}\left(L_{y}=4\right), F B C_{y},(T) P B C_{x}\right) \simeq 3.228126
$$

Thus, for the triangular strips with $\left(F B C_{y},(T) P B C_{x}\right)$ boundary conditions for which the chromatic polynomials have been calculated so far, i.e. for the widths $L_{y}=2,3$, and $4, q_{c}$ 


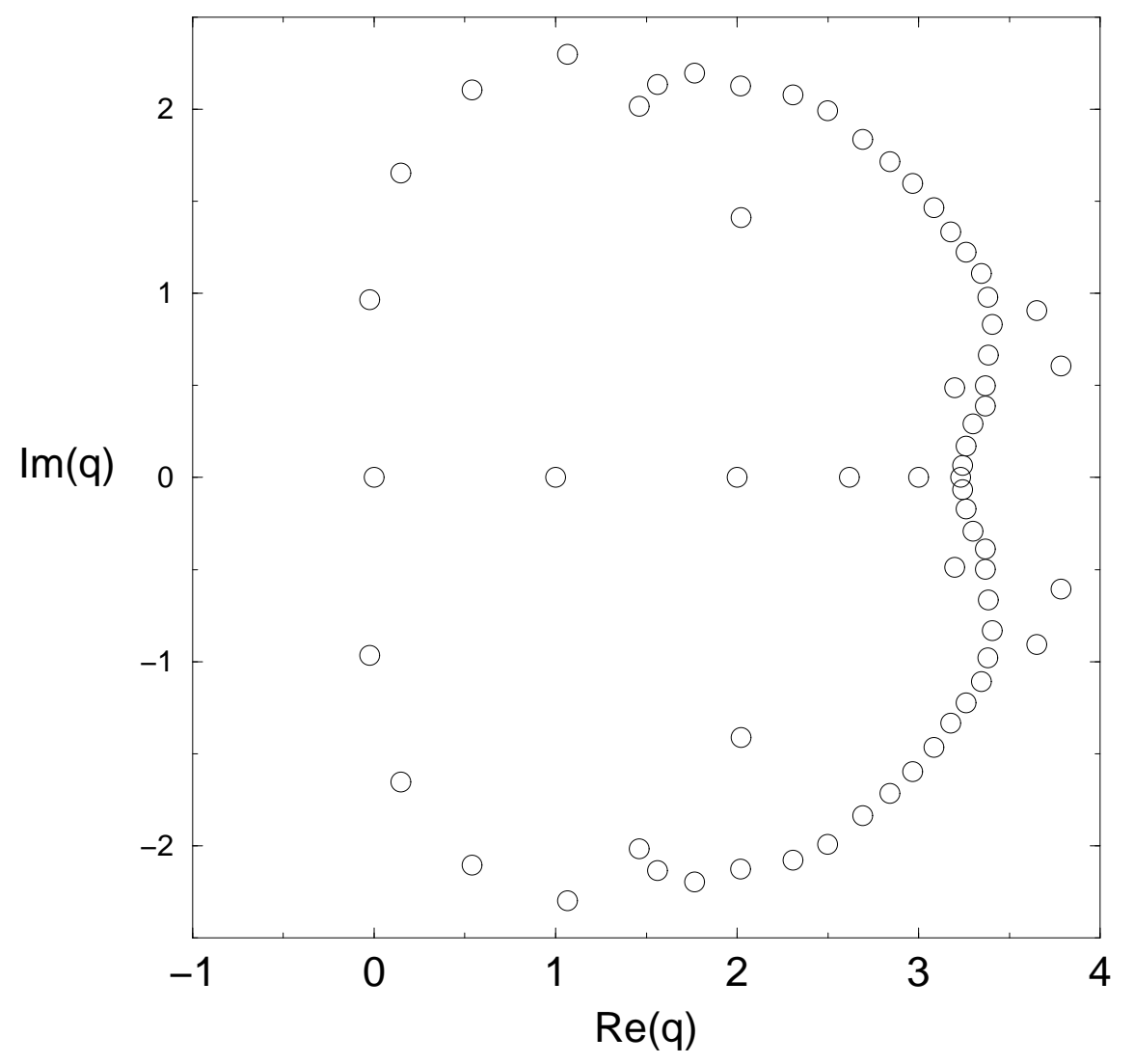

Figure 3: $\mathcal{B}$ and chromatic zeros for the $L_{y}=4, L_{x}=m=16$ (i.e., $n=64$ ) cyclic triangular strip graph. 
is a nondecreasing function of $L_{y}: q_{c}(\operatorname{tri}(2))=3, q_{c}(\operatorname{tri}(3))=3$, and $q_{c}(\operatorname{tri}(4))=3.228 \ldots$ We have found that this is also true for the strips of the square lattice with the same cyclic or Möbius boundary conditions: $q(s q(1))=q_{c}(s q(2))=2, q_{c}(s q(3)) \simeq 2.34$, and $q_{c}(s q(4)) \simeq 2.49$. This contrasts with the non-monotonic behavior of $q_{c}$ as a function of $L_{y}$ that we have found for strips with $\left(P B C_{y}, F B C_{x}\right)$ boundary conditions (see eq. (5.16) below). It is anticipated that for cyclic and Möbius strips of the triangular lattice, as $L_{y}$ increases beyond $3, q_{c}$ will approach the $2 \mathrm{D}$ value $q_{c}(\operatorname{tr} i)=4$.

Another important feature of the locus $\mathcal{B}$ for the $L_{y}=4$ cyclic triangular strip, which is the same as was true of the $L_{y}=2$ and $L_{y}=3$ cyclic triangular strips, is that it crosses the real axis at the points $q=0,2$ and 3 . This leads us to the conjecture

$$
\text { Conjecture: } \mathcal{B} \supset\{q=0,2,3\} \text { for } \operatorname{tri}\left(L_{y}, F B C_{y},(T) P B C_{x}\right) \quad \forall L_{y} \geq 2
$$

The crossing at $q=0$ is present in a very wide class of families of graphs that contain global circuits 23.

The crossing at $q=2$ that we have found for $L_{y}=2,3,4$ signals a zero-temperature critical point of the Ising antiferromagnet on these triangular strips, and similarly for the generalization (3.8) to arbitrary $L_{y}$. Note that this $T=0$ critical point involves frustration, since not all of the spin-spin interactions around a triangle can have their energies minimized. Frustration is also responsible for the $T=0$ critical point of the Ising antiferromagnet on the full 2D triangular lattice [38, 39], but the nature of the critical singularities in the free energy, correlation length, etc. are different: they are algebraic for the triangular lattice, but are essential singularities for the $L_{y} \times \infty$ strips [40]. As will be shown below, this crossing of $\mathcal{B}$ at $q=2$ is also found for the $L_{y}=2$ strips with torus or Klein bottle boundary conditions, i.e. $\left(P B C_{y}, P B C_{x}\right)$ or $\left(P B C_{y}, T P B C_{x}\right)$. Thus, it appears to be a general feature of the strip graphs of the triangular lattice with periodic or twisted periodic longitudinal boundary conditions. Indeed, the respective boundaries $\mathcal{B}$ for the $L_{y}=2$ cyclic and Möbius strips of the square, triangular, and kagomé lattices, and for the $L_{y}=3$ cyclic and Möbius strips of the square lattice [11] and the torus and Klein bottle strips of the square lattice [26] also cross the real $q$ axis at $q=2$ (as well as $q=0$ ).

In contrast, for strips with $\left(F B C_{y}, F B C_{x}\right)$ (open) or $\left(P B C_{y}, F B C_{x}\right)$ (cylindrical) boundary conditions, $\mathcal{B}$ does not pass through $q=0$ and, while $\mathcal{B}$ for the $L_{y}=3$ open square strip crosses the real axis at $q=2$, the respective loci $\mathcal{B}$ for the $L_{y}=4,5$ open and cylindrical square strips and the open and cylindrical triangular strips with $L_{y}=3,4,5$ do not, as discussed further below.

In regions $R_{j}, 1 \leq j \leq 4$, the dominant terms are, respectively, the root of maximal magnitude of (1) the first quartic equation (9.2.1), the second quartic equation (9.2.2), (3) 
the eighth-degree equation (9.2.7), (4) the ninth-degree equation (9.2.16). In region $R_{1}$, we label the dominant term as $\lambda_{t 4, R 1}$, and so forth for the other regions. In the complexconjugate regions $R_{5}, R_{5}^{*}$ the dominant term is the largest-magnitude root of the ninth-degree equation (9.2.16), which we label as $\lambda_{t 4, R 5}$. We have

$$
\begin{gathered}
W=\left(\lambda_{t 4, R 1}\right)^{1 / 4}, \quad q \in R_{1} \\
|W|=\left|\lambda_{t 4, R j}\right|^{1 / 4}, \quad q \in R_{j}, j=2,3,4
\end{gathered}
$$

and

$$
|W|=\left|\lambda_{t 4, R 5}\right|^{1 / 4}, \quad q \in R_{5}, R_{5}^{*} .
$$

As usual, the boundaries of the regions are defined by the degeneracies, in magnitude, of the leading $\lambda_{t 4, j}$ 's in these regions. For example, on the real axis, one has $\left|\lambda_{t 4, R 1}\right|=\left|\lambda_{t 4, R 2}\right|$ at $q_{c},\left|\lambda_{t 4, R 2}\right|=\left|\lambda_{t 4, R 3}\right|$ at $q=3,\left|\lambda_{t 4, R 3}\right|=\left|\lambda_{t 4, R 4}\right|$ at $q=2$, and $\left|\lambda_{t 4, R 4}\right|=\left|\lambda_{t 4, R 1}\right|$ at $q=0$.

In contrast to the situation for the $L_{y}=2$ and $L_{y}=3$ cyclic strips of the triangular lattice, here $q_{c}$ is a regular instead of a multiple (= intersection) point on the algebraic curve forming $\mathcal{B}$, in the terminology of algebraic geometry (a multiple point on an algebraic curve is a point where several branches of the curve cross each other).

Another qualitative difference is that for $L_{y}=2$ and $L_{y}=3, \mathcal{B}$ contained support only

for $\operatorname{Re}(q) \geq 0$, and the only point on $\mathcal{B}$ with $\operatorname{Re}(q)=0$ was the origin itself; however, for $L_{y}=4, \mathcal{B}$ extends slightly into the half-plane with $\operatorname{Re}(q)<0$. Although these lattice strips involve global circuits, it was found in [19] that this is not a necessary condition for chromatic zeros and loci $\mathcal{B}$ of lattice strips (or homeomorphic expansions thereof) to have support for $\operatorname{Re}(q)<0$; this was also evident in results of [29], ruling out a conjecture in [17]. In the case of strips of the square lattice we had found that for $L_{y}=1$ and $2, \mathcal{B}$ only had support for $\operatorname{Re}(q) \geq 0$, but for $L_{y}=3$ and $L_{y}=4$ it had support also for $\operatorname{Re}(q)<0$.

Finally, another general feature is that the motion of the outermost curves forming part of $\mathcal{B}$ for $L_{y}=2,3,4$ is consistent with the inference that as $L_{y}$ increases, these form a limiting curve.

\section{$4 L_{y}=3$ Triangular Lattice Strips with Torus and Klein Bottle Boundary Conditions}

For the $L_{y}=3$ triangular lattice strips with torus boundary conditions (denoted with the subscript $t t$ ), we calculate, by the same methods as above,

$$
P\left(\operatorname{tri}\left(L_{y}=3\right)_{m}, P B C_{y}, P B C_{x}, q\right)=\sum_{j=1}^{11} c_{t t, j}\left(\lambda_{t t, j}\right)^{m}
$$


where

$$
\begin{gathered}
\lambda_{t t, 1}=-2, \quad c_{t t, 1}=\frac{1}{3}(q-1)\left(q^{2}-5 q+3\right) \\
\lambda_{t t, 2}=q-2, \quad c_{t t, 2}=\frac{1}{2}(q-1)(q-2) \\
\lambda_{t t, 3}=3 q-14, \quad c_{t t, 3}=\frac{1}{2} q(q-3), \\
\lambda_{t t, 4}=-2(q-4)^{2}, \quad c_{t t, 4}=q-1, \\
\lambda_{t t, 5}=q^{3}-9 q^{2}+29 q-32, \quad c_{t t, 5}=1 \\
\lambda_{t t,(6,7)}=e^{ \pm 2 \pi i / 3}, \quad c_{t t, 6}=c_{t t, 7}=\frac{1}{6} q(q-1)(2 q-7) \\
\lambda_{t t,(8,9)}=\left(q^{2}-5 q+7\right) e^{ \pm 2 \pi i / 3}, \quad c_{t t, 8}=c_{t t, 9}=q-1 \\
\lambda_{t t,(10,11))}=-(2 q-7) e^{ \pm 2 \pi i / 3}, \quad c_{t t, 10}=c_{t t, 11}=\frac{1}{2}(q-1)(q-2) .
\end{gathered}
$$

Note that although several of the coefficients are complex (and have coefficients that are not integers), the chromatic polynomial itself is, of course, a polynomial with integer coefficients. The sum of the complex terms can be written as

$$
\sum_{j=6}^{11} c_{t t, j}\left(\lambda_{t t, j}\right)^{m}=(q-1)\left[\frac{q(2 q-7)}{3}+2\left(q^{2}-5 q+7\right)^{m}+(q-2)[-(2 q-7)]^{m}\right] \cos \left(\frac{2 m \pi}{3}\right)
$$

None of these terms with $6 \leq j \leq 11$ can be dominant anywhere in the $q$ plane since this would imply that the limit (1.2) would not exist. As compared with previously calculated strip graphs, this exhibits a number of qualitatively new features: (i) previously, only one $\lambda_{j}$ was a constant, independent of $q$, and this was always equal to either 1 or -1 ; here there are three, and none of these is equal to \pm 1 ; (ii) previously, all of the $\lambda_{j}$ 's were either polynomials with real coefficients or algebraic functions of polynomials with real coefficients. By general arguments given before [23], the dominant $\lambda_{j}$ in region $R_{1}$ has as its highest power $q^{n / m}$, i.e., in the present case, $q^{3}$, and has coefficient 1 . We find that the generating function for this case has $\operatorname{deg}_{x}(\mathcal{N})=9$ and $\operatorname{deg}_{x}(\mathcal{D})=11$, so that all of the $\lambda_{j}$ 's in $\mathcal{D}$ contribute to $P$. 
Given that $\chi=4$ for $m=1$ or $2 \bmod 3$ from eq. (1.5), it follows that for these $m$ values, and $q=3, P\left(\operatorname{tri}\left(L_{y}=3\right)_{m}, P B C_{y}, P B C_{x}, q=3\right)=0$; however, $\chi=3$ for $m=0 \bmod 3$; in this case, we find (for $m \geq 3$ )

$$
P\left(\operatorname{tri}\left(L_{y}=3\right)_{m}, P B C_{y}, P B C_{x}, q=3\right)=6 \quad \text { for } \quad m=0 \quad \bmod 3
$$

This is analogous to eq. (2.26) above.

For the $L_{y}=3$ triangular lattice strips with Klein bottle topology (denoted with the subscript $t k$ ) we calculate

$$
\begin{aligned}
& P\left(\operatorname{tri}\left(L_{y}=3\right)_{m}, P B C_{y}, T P B C_{x}, q\right)=\sum_{j=1}^{5} c_{t k, j}\left(\lambda_{t k, j}\right)^{m} \\
& =-(q-1)(-2)^{m}-\frac{1}{2}(q-1)(q-2)(q-2)^{m}+\frac{1}{2} q(q-3)(3 q-14)^{m}+ \\
& (q-1)\left[-2(q-4)^{2}\right]^{m}+\left(q^{3}-9 q^{2}+29 q-32\right)^{m} .
\end{aligned}
$$

Thus, $c_{t k, j}=c_{t t, j}, j=1, . .5$, and the six $\lambda_{t t, j}, j=6, . .11$ that involve complex factors do not contribute to $P\left(\operatorname{tri}\left(L_{y}=3\right)_{m}, P B C_{y}, T P B C_{x}, q\right)$. Since none of these six $\lambda_{t t, j}$ 's is dominant anywhere, it follows that $\mathcal{B}$ is the same for the $L_{y}=3$ triangular lattice strips with torus and Klein bottle topology, just as was true of the analogous square lattice strips [26], and in accord with the general discussion of Ref. [23]. For this case the generating function has $\operatorname{deg}_{x}(\mathcal{N})=2$ and $\operatorname{deg}_{x}(\mathcal{D})=5$, so that, as was true of the $L_{y}=3$ Möbius and torus strips of the triangular lattice, all of the $\lambda_{j}$ 's in $\mathcal{D}$ contribute to $P$.

For the sum of the coefficients we find, for the triangular $L_{y}=3$ strip with torus boundary conditions

$$
C=P\left(K_{3}, q\right)=q(q-1)(q-2) \quad \text { for } \quad G=\operatorname{tri}\left(L_{y}=3\right) \quad \text { with } \quad\left(P B C_{y}, P B C_{x}\right)
$$

and for this strip with Klein bottle boundary conditions

$$
C=0 \quad \text { for } \quad G=\operatorname{tri}\left(L_{y}=3\right) \quad \text { with } \quad\left(P B C_{y}, T P B C_{x}\right)
$$

These are the same respective values as those calculated in [26] for the corresponding $L_{y}=3$ strips of the square lattice with torus and Klein bottle boundary conditions.

Chromatic zeros for the $L_{y}=3, L_{x}=m=20$ torus graph of the triangular lattice are

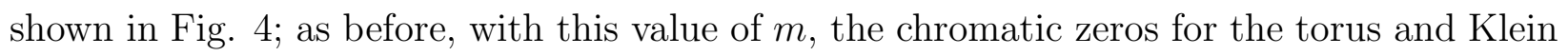
bottle boundary conditions are generally similar and both sets of chromatic zeros lie close 


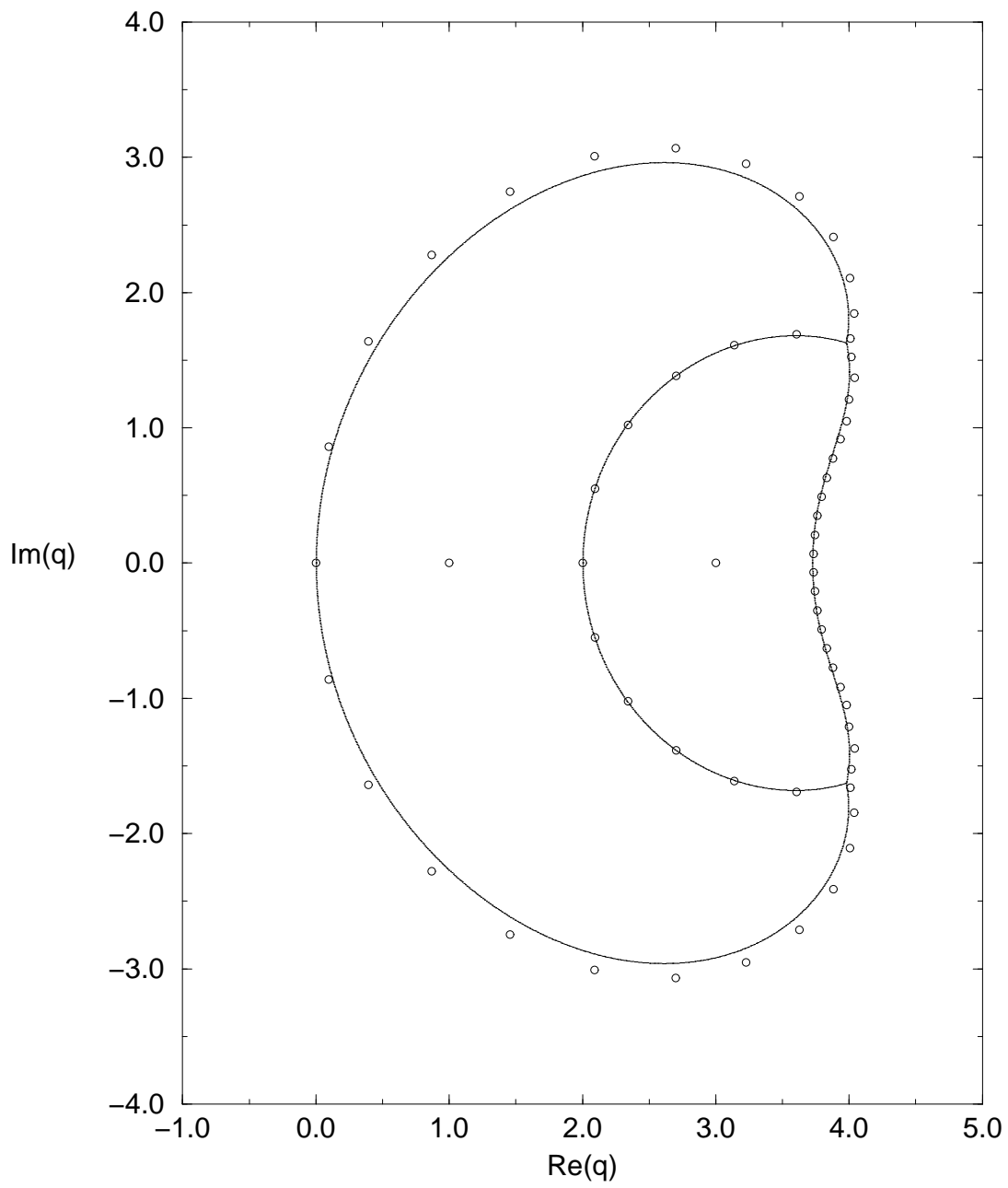

Figure 4: $\mathcal{B}$ and chromatic zeros for the $L_{y}=3, L_{x}=m=20$ (i.e., $n=60$ ) triangular strip graph with torus boundary conditions. 
to the boundary $\mathcal{B}$ and indicate its position. The locus $\mathcal{B}$ and the $W$ functions are the same for the torus and Klein bottle graph families. We find

$$
q_{c} \simeq 3.7240756 \ldots \text { for } \quad\{G\}=\operatorname{tri}\left(L_{y}=3\right) \text { with } \quad\left(P B C_{y}, P B C_{x}\right) \text { or }\left(P B C_{y}, T P B C_{x}\right)
$$

This value of $q_{c}$ is given by the real root of the degeneracy equation $q^{3}-9 q^{2}+32 q-46=0$. It is interesting that this is just $7 \%$ below the value for the infinite $2 \mathrm{D}$ triangular lattice, $q_{c}($ tri $)=4$. Thus, for the width $L_{y}=3$ strips where a comparison can be made, the use of both periodic transverse boundary conditions and periodic or twisted periodic longitudinal boundary conditions expedites the approach to the 2D thermodynamic limit, in the sense that it yields a value of $q_{c}$ that is substantially closer to the $2 \mathrm{D}$ value than was obtained for the same width strip with free transverse boundary conditions in eq. (2.40). This is expected since for the torus or Klein bottle boundary conditions (a) the resultant graphs are $\Delta$-regular with the degree (coordination number) $\Delta=6$ of the $2 \mathrm{D}$ triangular lattice, and (b) there are no boundaries to the surface on which the graphs are embedded. In contrast, for the boundary conditions of types (i)-(iv) (aside from degenerate cases) the graphs are not $\Delta$-regular and they do have boundaries.

The locus $\mathcal{B}$ has support for $\operatorname{Re}(q) \geq 0$ and separates the $q$ plane into three regions. The outermost one, region $R_{1}$, extends to infinite $|q|$ and includes the intervals $q \geq q_{c}$ and $q \leq 0$ on the real $q$ axis. Region $R_{2}$ includes the real interval $2 \leq q \leq q_{c}$ and extends upward and downward to the complex conjugate triple points on $\mathcal{B}$ at $q_{t}, q_{t}^{*} \simeq 4.0 \pm 1.7 i$. Region $R_{3}$ is the innermost one and includes the real interval $0 \leq q \leq 2$. The maximum value of $|\operatorname{Im}(q)|$ on the boundary between $R_{1}$ and $R_{3}$ is about 3.0, occurring at $R e(q) \simeq 2.6$. The maximum value of $|q|$ on $\mathcal{B}$ is roughly 4.6 , occurring at $q \simeq 3.9 \pm 2.5 i$. The boundary between $R_{2}$ and $R_{3}$ curves to the right as one increases $|\operatorname{Im}(q)|$, extending from $q=2$ upward to $q_{t}$ and downward to $q_{t}^{*}$. As is evident in Fig. 4 , the density of chromatic zeros is highest on the $R_{1}-R_{2}$ boundary near $q_{c}$. The feature that $\mathcal{B}$ has support only for $\operatorname{Re}(q) \geq 0$ is the same as was found [26] for the $L_{y}=3$ strips of the square lattice with torus and Klein bottle topology.

In region $R_{1}, \lambda_{t t, 5}=\lambda_{t k, 5}$ is the dominant $\lambda_{j}$, so

$$
W=\left(q^{3}-9 q^{2}+29 q-32\right)^{1 / 3}, \quad q \in R_{1} .
$$

The fact that this is the same as $W$ for the $\left(\mathrm{PBC}_{y}, \mathrm{FBC}_{x}\right)$ case, eq. (5.3), is a general result [23. The importance of the $\mathrm{PBC}_{y}$ is evident from the fact that for the same width of three squares, the strip with $\left(\mathrm{FBC}_{y}, \mathrm{FBC}_{x}\right)$ yields a different $W$ 17.

\footnotetext{
${ }^{8} \mathrm{~A} \Delta$-regular graph is a graph all of whose vertices have the same degree, $\Delta$, where the degree of a vertex is defined as the number of edges connected to it.
} 
In region $R_{2} \lambda_{t t, 3}=\lambda_{t k, 3}$ is dominant, so

$$
|W|=|3 q-14|^{1 / 3}, \quad q \in R_{2}
$$

(in regions other than $R_{1}$, only $|W|$ can be determined unambiguously [7]). In region $R_{3}$, $\lambda_{t t, 4}=\lambda_{t k, 4}$ is dominant, so

$$
|W|=\left|2(q-4)^{2}\right|^{1 / 3}, \quad q \in R_{3} .
$$

At all of the three points, $q=0,2$, and $q=q_{c} \simeq 3.72$ where $\mathcal{B}$ crosses the real $q$ axis, it does so vertically. The present results are in accord with the inference [17, 11] that for a recursive graph with regular lattice structure, a sufficient condition for $\mathcal{B}$ to separate the $q$ plane into two or more regions is that it contains a global circuit, i.e. a path along a lattice direction whose length goes to infinity as $n \rightarrow \infty$; here this is equivalent to $\mathrm{PBC}_{x}$.

Our calculations of the zero-temperature Potts antiferromagnet partition functions (chromatic polynomials) and exponential of the entropy, $W$, for $L_{y}=3$ strips of the triangular lattice with various boundary conditions including free and periodic or reversed-orientation periodic thus elucidate the role that these boundary conditions and the associated topologies play. One particular feature of note is that the torus and Klein bottle graphs have interestingly different chromatic polynomials, with different $N_{\lambda}$, but the $W$ functions and hence the boundaries $\mathcal{B}$ are the same.

\section{Width $L_{y}=5,6$ Strips of the Triangular Lattice with $\left(P B C_{y}, F B C_{x}\right)$}

We first briefly review the cases $L_{y}=3,4$ [18, 20, 23]. The chromatic polynomial for the $L_{y}=3$ triangular $(t)$ strip with $n=3(m+2)$ vertices (following the labelling conventions in [17]) and $\left(P B C_{y}, F B C_{x}\right)$ boundary conditions, denoted $t 3 P F$ in the subscripts, has $N_{\lambda}=1$,

$$
\begin{gathered}
\lambda_{t 3 P F}=q^{3}-9 q^{2}+29 q-32, \\
P\left(\operatorname{tri}\left(3 \times m, P B C_{y}, F B C_{x}\right), q\right)=q(q-1)(q-2)\left(\lambda_{t 3 P F}\right)^{m+1},
\end{gathered}
$$

and

$$
W\left(\operatorname{tri}\left(L_{y}=3\right), P B C_{y}, F B C_{x}, q\right)=\left(q^{3}-9 q^{2}+29 q-32\right)^{1 / 3}
$$

with $\mathcal{B}=\emptyset$. 
For the $L_{y}=4$ strip with $\left(P B C_{y}, F B C_{x}\right)$ boundary conditions and $n=4(m+2)$ vertices, one had [18, 20] $N_{\lambda}=2$ and

$$
P\left(\operatorname{tri}\left(4 \times m, P B C_{y}, F B C_{x}\right), q\right)=\sum_{j=1}^{2} c_{t 4 P F, j}\left(\lambda_{t 4 P F, j}\right)^{m+1}
$$

where

$$
\lambda_{t 4 P F, j}=\frac{(q-3)}{2}\left[T_{t 4 P F} \pm(q-4) \sqrt{R_{t 4 P F}}\right]
$$

where

$$
\begin{gathered}
T_{t 4 P F}=q^{3}-9 q^{2}+33 q-48 \\
R_{t 4 P F}=q^{4}-10 q^{3}+43 q^{2}-106 q+129 .
\end{gathered}
$$

In this case, as is evident in Fig. 4(a) of $[18]), \mathcal{B}$ includes both arcs and a closed oval invariant under complex conjugation which crossed the real axis where the prefactor of the square root vanished, i.e. at $q_{c}=4$, and at the real root of $T_{t 4 P F}$, namely $q \simeq 3.481406$. There are two regions, $R_{1}$ and $R_{2}$, the exterior and interior of the oval. We have

$$
W\left(\operatorname{tri}, 4 \times \infty, P B C_{y}, q\right)=\left(\lambda_{t 4 P F, 1}\right)^{1 / 4}, \quad q \in R_{1}
$$

and

$$
W\left(t r i, 4 \times \infty, P B C_{y}, q\right)=\left(\lambda_{t 4 P F, 2}\right)^{1 / 4}, \quad q \in R_{2}
$$

with appropriate choices of branch cuts.

We have calculated new results for the width $L_{y}=5$ and $L_{y}=6$ strips with $\left(P B C_{y}, F B C_{x}\right)$ boundary conditions and $n=L_{y}(m+2)$ vertices. For $L_{y}=5$ we find $N_{\lambda}=2$ and

$$
P\left(\operatorname{tri}\left(5 \times m, P B C_{y}, F B C_{x}\right), q\right)=\sum_{j=1}^{2} c_{t 5 P F, j}\left(\lambda_{t 5 P F, j}\right)^{m+1}
$$

where

$$
\lambda_{t 5 P F, j}=\frac{1}{2}\left[T_{t 5 P F} \pm S_{t 5 P F} \sqrt{R_{t 5 P F}}\right]
$$

where

$$
\begin{gathered}
T_{t 5 P F}=q^{5}-15 q^{4}+98 q^{3}-355 q^{2}+711 q-614 \\
R_{t 5 P F}=q^{4}-8 q^{3}+26 q^{2}-60 q+85
\end{gathered}
$$

and

$$
S_{t 5 P F}=q^{3}-11 q^{2}+43 q-58
$$


As before, the coefficients $c_{t 5 P F, j}$ can be computed using eq. (2.9) in terms of the generating function. This generating function is given in the appendix.

Just as was true for the $L_{y}=4$ triangular lattice strip with $\left(P B C_{y}, F B C_{x}\right)$, in the present $L_{y}=5$ case $\mathcal{B}$ includes both a pair of complex conjugate arcs and an oval that crosses the real axis at two points. Analogously to the previous case, one of these points is the real zero of $T_{t 5 P F}$ at

$$
q_{\ell} \simeq 3.207224
$$

and the other is the real zero of the prefactor $S_{t 5 P F}$ in front of the square root, at

$$
q=3.284775=q_{c} \quad \text { for } \quad\{G\}=\operatorname{tri}\left(5 \times \infty, P B C_{y}, F B C_{x}\right) .
$$

Taking the approximate center of the oval as the average of the left and right crossings, $q_{\text {center }}=\left(q_{\ell}+q_{c}\right)$, one finds that

$$
q_{\text {center }} \simeq 3.2460
$$

near to the Tutte-Beraha number $B_{7} \simeq 3.246980 \ldots$, where 4 , 14

$$
B_{r}=2+2 \cos \left(\frac{2 \pi}{r}\right) \text {. }
$$

Chromatic zeros near $B_{7}$ were also noted in [29] for these types of strips. Note that $q_{c}$ decreases from 4 to the above value in eq. (5.16) as $L_{y}$ increases from 4 to 5 . Hence, in contrast to our calculations of strips of the square and triangular lattices with $\left(F B C_{y}, F B C_{x}\right)$, $\left(F B C_{y},(T) P B C_{x}\right)$, and $\left(P B C_{y},(T) P B C_{x}\right)$, where in all cases considered, the respective $q_{c}$ 's were nondecreasing functions of $L_{y}$, here we find that this is not the case for boundary conditions of the type $\left(P B C_{y}, F B C_{x}\right)$.

Chromatic zeros and $\mathcal{B}$ for this strip are shown in Fig. 5; $\mathcal{B}$ separates the $q$ plane into two regions, $R_{1}$, the exterior, and $R_{2}$, the interior, of the oval shown in the figure. Comparing the ovals for the $L_{y}=4$ strip (Fig. 4(a) of [18]) and the $L_{y}=5$ strip (Fig. 5 here), one sees that the oval shrinks in size. We have

$$
W\left(\operatorname{tri}, 5 \times \infty, P B C_{y}, q\right)=\left(\lambda_{t 5 P F, 1}\right)^{1 / 5}, \quad q \in R_{1}
$$

and

$$
W\left(\text { tri }, 5 \times \infty, P B C_{y}, q\right)=\left(\lambda_{t 5 P F, 2}\right)^{1 / 5}, \quad q \in R_{2}
$$

with appropriate choices of branch cuts.

For the strip of with $L_{y}=6$ and $\left(P B C_{y}, F B C_{x}\right)$ we find $N_{\lambda}=5$ and

$$
P\left(\operatorname{tri}\left(6 \times m, P B C_{y}, F B C_{x}\right), q\right)=\sum_{j=1}^{5} c_{t 6 P F, j}\left(\lambda_{t 6 P F, j}\right)^{m+1}
$$




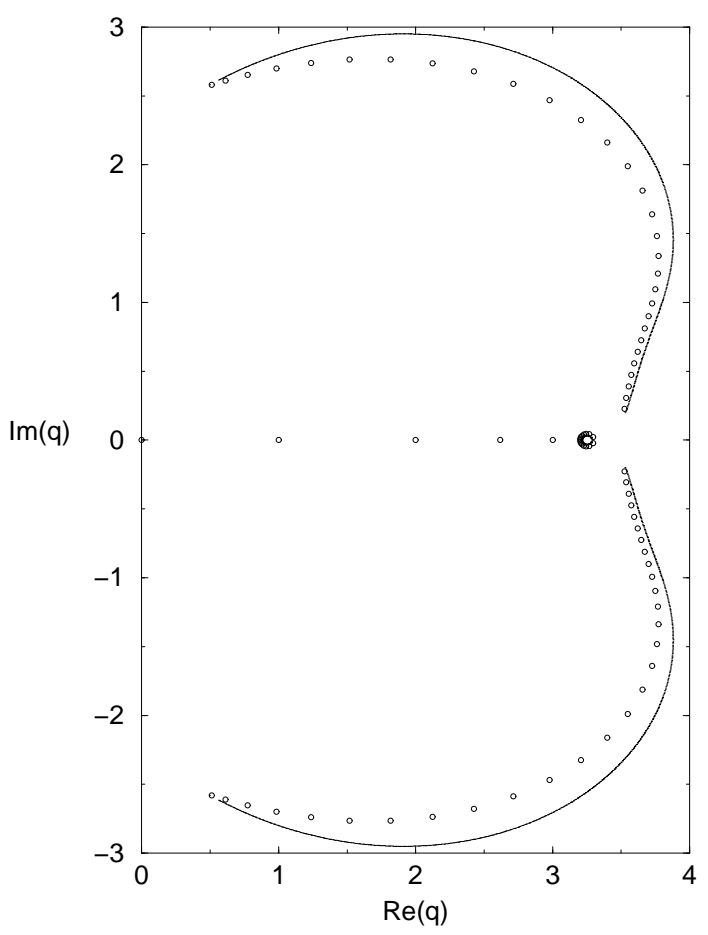

Figure 5: Locus $\mathcal{B}$ for the width $L_{y}=5$ strip (tube) of the triangular lattice with $\left(P B C_{y}, F B C_{x}\right)$ boundary conditions. Thus, the cross sections of the tube form pentagons. For comparison, chromatic zeros calculated for the strip length $L_{x}=m+2=16$ (i.e., $n=80$ vertices) are shown. 
Here the $\lambda_{t 6 P F}$ 's are the roots of a fifth-order equation, so we cannot solve for them in terms of algebraic expressions as was possible for previous strips of this type with $L_{y}=3,4,5$. We give the generating function for this strip in the appendix.

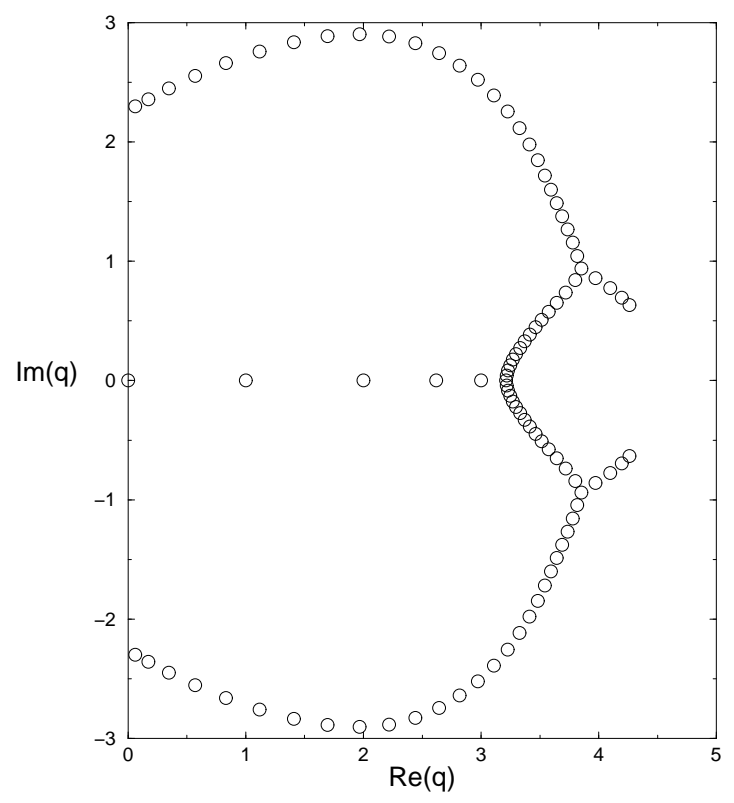

Figure 6: Locus $\mathcal{B}$ for the width $L_{y}=6$ strip (tube) of the triangular lattice with $\left(P B C_{y}, F B C_{x}\right)$ boundary conditions. Thus, the cross sections of the tube form hexagons. For comparison, chromatic zeros calculated for the strip length $L_{x}=m+2=16$ (i.e., $n=96$ vertices) are shown.

Chromatic zeros for this strip are shown in Fig. 6 for $L_{x}=m+2=16$, i.e., $n=96$; this length is sufficiently great that the chromatic zeros give an approximate indication of the location of the locus $\mathcal{B}$. The locus thereby inferred appears to consist of a single connected set of curves and crosses the real axis. From our exact analytic expressions, we calculate the this crossing point to be

$$
q_{c}=3.252419 \text { for } \quad\{G\}=\operatorname{tri}\left(6 \times \infty, P B C_{y}, F B C_{x}\right)
$$

The morphology of chromatic zeros for this long $6 \times 16$ cylindrical strip is similar to that found for a $8 \times 8$ patch with cylindrical boundary conditions in [29]. In both cases, one can figuratively think of how the inferred loci $\mathcal{B}$ can be modified to yield the locus for the $L_{y}=\infty$ strip with $\left(P B C_{y}, F B C_{x}\right)$ cylindrical boundary conditions: one (i) pulls the lefthand endpoints further to the left (into the $\operatorname{Re}(q)<0$ half-plane) and around so that they meet in a cusp at the origin, $q=0$; (ii) pulls the right-hand endpoints of the prongs over 
and around so that they meet in a cusp at $q=4$; (iii) shifts the crossing curve slightly to the right so that it crosses the real axis at $q \simeq 3.82$; and (iv) makes minor shifts of the rest of the locus $\mathcal{B}$ so as to obtain $\mathcal{B}$ for the cylindrical strip with $L_{y}=\infty$. In this respect, just as was discussed in [20], one sees that the periodic transverse boundary conditions help to minimize finite-size effects. Clearly, for a fixed $L_{y}$, with $L_{x} \rightarrow \infty$, finite-size effects are reduced the most for $\left(P B C_{y},(T) P B C_{x}\right)$, next-most for $\left(P B C_{y}, F B C_{x}\right)$ and $\left(F B C_{y},(T) P B C_{x}\right)$, and least for $\left(F B C_{y}, F B C_{x}\right)$. We find

$$
W\left(\text { tri, } 6 \times \infty, P B C_{y}, q\right)=\left(\lambda_{t 6 P F, \max }\right)^{1 / 6} \quad \text { for } q \in R_{1}
$$

where $\lambda_{t 6 P F, \max }$ denotes the root with the maximal magnitude.

It is of interest to use these results to study the approach of $W$ to the limit for the full infinite 2D triangular lattice, extending the work of [20]. In Table 1 we list the various values of $W\left(\operatorname{tri}\left(L_{y} \times \infty, P B C_{y}, F B C_{x}\right), q\right)$, denoted as $W\left(L_{y}, q\right)$ to save space, together with the corresponding values of $W$ for the full $2 \mathrm{D}$ triangular lattice, $W(\operatorname{tr} i, q)$, obtained via numerical evaluation of an infinite product representation in [29] as checked by series expansions and rigorous bounds [37], and the ratio $R_{W}\left(\operatorname{tri}\left(L_{y} \times \infty\right), P B C_{y}, F B C_{x}, q\right)$, where

$$
R_{W}\left(\operatorname{tri}\left(L_{y} \times \infty\right), B C_{y}, B C_{x}, q\right)=\frac{W\left(\operatorname{tri}\left(L_{y} \times \infty\right), B C_{y}, B C_{x}, q\right)}{W(\operatorname{tri}, q)} .
$$

This extends the previous study in 20]. Recall that for $q \geq 4, W\left(\operatorname{tri}\left(L_{y} \times \infty\right), B C_{y}, B C_{x}, q\right)$ is independent of $B C_{x}$ [20, 23]. Evidently, the approach of $W$ for the infinite-length finitewidth triangular strips to the $2 \mathrm{D}$ thermodynamic limit as $L_{y}$ increases is quite rapid; for moderate values of $q$, say 6 or 7 , the ratio $R_{W}\left(\operatorname{tr} i(5 \times \infty), P B C_{y}, F B C_{x}, q\right)$ is equal to 1 to within approximately $10^{-3}$ or better, and the ratio $R_{W}\left(\operatorname{tri}(6 \times \infty), P B C_{y}, F B C_{x}, q\right)$ is at least as close to 1 . As was proved in [20], the approach is non-monotonic for $P B C_{y}$ (and monotonic for $F B C_{y}$ ).

\section{Width $L_{y}=5$ Strips of the Triangular Lattices with $\left(F B C_{y}, F B C_{x}\right)$}

Previous studies have been published on strips of various lattices with $\left(F B C_{y}, F B C_{x}\right)$ boundary conditions [17, 18, 19. In the case of the the square and triangular lattice, these went up to $L_{y}=4$ and involved $\lambda_{j}$ 's that were roots of a cubic and quartic equation, respectively. Although one can calculate chromatic polynomials for wider strips, the analysis is more cumbersome if the equations defining the $\lambda_{j}$ 's are higher than quartic, so that one cannot solve for these $\lambda_{j}$ 's as analytic closed-form algebraic expressions. A study of these 
Table 1: Comparison of values of $W\left(\operatorname{tri}\left(L_{y}\right), P B C_{y}, q\right)$ with $W(\operatorname{tri}, q)$ for $4 \leq q \leq 10$ and $B C_{x}=F B C_{x}$ or (T) $P B C_{x}$. For each value of $q$, the quantities in the upper line are identified at the top and the quantities in the lower line are the values of $R_{W}\left(\operatorname{tri}\left(L_{y}\right), P B C_{y}, q\right)$. The $P B C_{y}$ is symbolized as $P_{y}$ in the table.

\begin{tabular}{|c|c|c|c|c|c|}
\hline \hline$q$ & $W\left(\operatorname{tri}(3), P_{y}, q\right)$ & $W\left(\operatorname{tri}(4), P_{y}, q\right)$ & $W\left(\operatorname{tri}(5), P_{y}, q\right)$ & $W\left(\operatorname{tri}(6), P_{y}, q\right)$ & $W(\operatorname{tri}, q)$ \\
\hline \hline 4 & 1.58740 & 1.18921 & 1.39252 & 1.49603 & 1.46100 \\
& 1.0865 & 0.8140 & 0.9531 & 1.0240 & 1 \\
\hline 5 & 2.35133 & 2.21336 & 2.26877 & 2.26894 & 2.26411 \\
& 1.0385 & 0.9776 & 1.0021 & 1.0021 & 1 \\
\hline 6 & 3.23961 & 3.185055 & 3.20718 & 3.20399 & 3.20388 \\
& 1.0112 & 0.9941 & 1.0010 & 1.0000 & 1 \\
\hline 7 & 4.17934 & 4.15965 & 4.16987 & 4.16805 & 4.16819 \\
& 1.0027 & 0.99795 & 1.0004 & 1.0000 & 1 \\
\hline 8 & 5.14256 & 5.13936 & 5.14446 & 5.14348 & 5.14358 \\
& 0.99980 & 0.9992 & 1.0002 & 1.0000 & 1 \\
\hline 9 & 6.11803 & 6.12324 & 6.12587 & 6.12533 & 6.12539 \\
& 0.99880 & 0.99965 & 1.0001 & 1.0000 & 1 \\
\hline 10 & 7.10059 & 7.11027 & 7.11161 & 7.11131 & 7.11134 \\
& 0.99849 & 0.99985 & 1.0000 & 1.0000 & 1 \\
\hline \hline
\end{tabular}

on wider open strips is in 41. As an illustration of this sort of situation, here we present a calculation of chromatic polynomials for the strip of the triangular lattice with width $L_{y}=5$ and $\left(F B C_{y}, F B C_{x}\right)$. For this width, the $\lambda_{j}$ 's are solutions of a degree-9 equation and hence cannot be solved for as algebraic roots. The method of calculation is again the iterated use of the deletion-contraction theorem. In [17], a given strip $\left(G_{s}\right)_{m}$ was constructed by $m$ successive additions of a subgraph $H$ to an endgraph $I$; here, $I=H$, so that, following the notation of [17, the total length of the strip graph $\left(G_{s}\right)_{m}$ is $m+2$ vertices, or equivalently, $m+1$ edges in the longitudinal direction. The results are conveniently expressed in terms of the coefficient functions in the generating function, as discussed above. For the width $L_{y}=5$ open strip of the triangular lattice we find $\operatorname{deg}_{z}(\mathcal{D})=N_{\lambda}=9$. The coefficient functions $b_{t 5 F F, j}$ and $A_{t 5 F F, j}$ (cf. eqs. (2.4) and (2.3)) are listed in the Appendix, where $F F$ is short for $\left(F B C_{y}, F B C_{x}\right)$.

In Fig. 7 we show a plot of chromatic zeros for the open strip of the triangular lattice with $L_{y}=5$ and length $m+2=16$ vertices, so that the strip has $n=80$ vertices in all. From an analysis of the degeneracy of leading $\lambda_{j}$ 's, we find the exact result

$$
q_{c}=3 \text { for } s q\left(5 \times \infty, F B C_{y}, F B C_{x}\right) .
$$




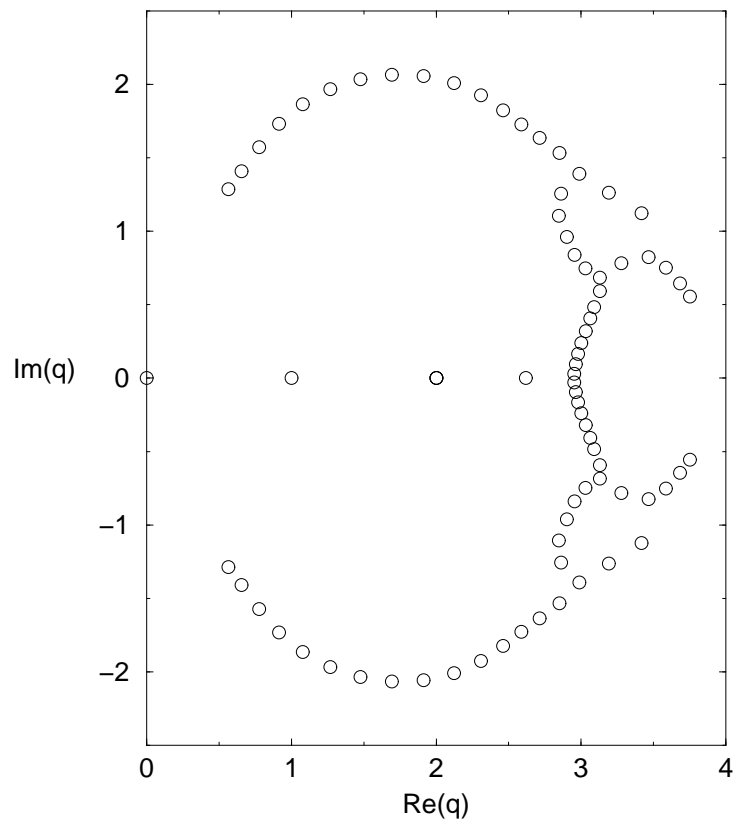

Figure 7: Chromatic zeros for the $L_{y}=5$ open strip of the triangular lattice of length $m+2=16$ vertices (i.e. total number of vertices $n=80$ ). 
This is in agreement with the chromatic zeros for the finite strip shown in Fig. 7. Again, we can compare Fig. 7 with the corresponding plots for $L_{y}=2$ and $L_{y}=3$ (Fig. 5(a,b) of [17]), and, just as was true for the corresponding three open strips of the square lattice, this comparison shows that as $L_{y}$ increases, the arcs forming $\mathcal{B}$ elongate and the arc endpoints nearest to the origin approach more closely to the origin. We recall that for the $L_{y}=4$ open triangular strip, no $q_{c}$ is defined since $\mathcal{B}$ does not cross the real axis.

For $q>q_{c}$, we have, for the physical ground state degeneracy per site of the $q$-state Potts antiferromagnet,

$$
W\left(t\left(5 \times \infty, F B C_{x}, F B C_{y}\right), q\right)=\left(\lambda_{t r i, j, \max }\right)^{1 / 5}
$$

where $\lambda_{s q, j, \max }$ denotes the solution of eq. (2.8) with the coefficients (9.4.1)-(9.4.9) that has the maximal magnitude in region $R_{1}$.

As before, it is of interest to use this result to study the approach of $W$ to the limit for the full infinite 2D triangular lattice, extending the work of [20] for this set of boundary conditions. In Table 2 we list the various values of $W\left(\operatorname{tri}\left(L_{y} \times \infty, F B C_{y}, B C_{x}\right), q\right)$, denoted as $W\left(L_{y}, q\right)$ to save space, together with the corresponding values of $W$ for the full $2 \mathrm{D}$ triangular lattice, $W(\operatorname{tr} i, q)$ and the ratio $R_{W}\left(\operatorname{tri}\left(L_{y} \times \infty\right), F B C_{y}, B C_{x}, q\right)=W\left(\operatorname{tri}\left(L_{y} \times\right.\right.$ $\left.\infty), F B C_{y}, B C_{x}, q\right) / W(\operatorname{tr} i, q)$. Recall that the value of $W$ for $q \geq 4$ is independent of $B C_{x}$ [20]. Again, the approach of $W$ for the infinite-length finite-width triangular strips to the 2D thermodynamic limit as $L_{y}$ increases is quite rapid.

\section{Comparative Discussion}

In this section we give a general discussion of the locus $\mathcal{B}$. We have found several interesting features:

1. For the strips of the triangular lattice of width $L_{y}=3$ and $L_{y}=4$ with $\left(F B C_{y},(T) P B C_{x}\right)$ and strips of width $L_{y}=3$ and $\left(P B C_{y},(T) P B C_{x}\right)$ boundary conditions studied here, we have shown that the locus $\mathcal{B}$ encloses regions of the $q$ plane including certain intervals on the real axis and passes through $q=0$ and 2 as well as other possible points, depending on the family. This extends the previous study of the $L_{y}=2$ strip of the triangular lattice with $\left(F B C_{y},(T) P B C_{x}\right)$ [1]. While the strips with $\left(F B C_{y},(T) P B C_{x}\right)$ have a locus $\mathcal{B}$ that passes through $q=3$, this is not the case with at least the $L_{y}=3$ strip with $\left(P B C_{y},(T) P B C_{x}\right)$. For the strips with $\left(F B C_{y},(T) P B C_{x}\right)$ considered here we have shown that $q_{c}$ is a nondecreasing function of $L_{y}$. As one increases $L_{y}$, the $L_{y}=4$ cyclic strip is the first one for which one can no longer solve for all of the $\lambda_{j}$ 's as algebraic roots, since some of the equations involved are higher than quartic. 
Table 2: Comparison of values of $W\left(\operatorname{tri}\left(L_{y} \times \infty\right), q\right)$ for $F B C_{y}$ and $B C_{x}=F B C_{x}$ or $(T) P B C_{x}$ with $W(\operatorname{tri}, q) \equiv W(\operatorname{tri}(\infty \times \infty), q)$ for $3 \leq q \leq 10$. For brevity of notation, we omit the $\left(F B C_{y}, B C_{x}\right)$ in the notation. For each value of $q$, the quantities in the upper line are identified at the top and the quantities in the lower line are the values of $R_{W}\left(\operatorname{tri}\left(L_{y}\right), q\right)$.

\begin{tabular}{|c|c|c|c|c|c|}
\hline$q$ & $\bar{W} W(\operatorname{tri}(2), q)$ & $\bar{W} W(\operatorname{tri}(3), q)$ & $\overline{\overline{W(\operatorname{tri}(4), q)}}$ & 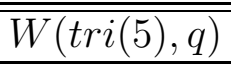 & $\bar{W} W(\operatorname{tri}(\infty), q)$ \\
\hline 4 & $\begin{array}{c}2 \\
1.369\end{array}$ & $\begin{array}{c}1.77173 \\
1.213\end{array}$ & $\begin{array}{c}1.67619 \\
1.147\end{array}$ & $\begin{array}{c}1.62270 \\
1.111\end{array}$ & $\begin{array}{c}1.46100 \\
1\end{array}$ \\
\hline 5 & $\begin{array}{c}3 \\
1.325\end{array}$ & $\begin{array}{c}2.72998 \\
1.206\end{array}$ & $\begin{array}{c}2.60495 \\
1.151\end{array}$ & $\begin{array}{c}2.53251 \\
1.1185\end{array}$ & $\begin{array}{c}2.26411 \\
1\end{array}$ \\
\hline 6 & $\begin{array}{c}4 \\
1.248\end{array}$ & $\begin{array}{c}3.71457 \\
1.159\end{array}$ & $\begin{array}{c}3.579715 \\
1.117\end{array}$ & $\begin{array}{c}3.50112 \\
1.093\end{array}$ & $\begin{array}{c}3.20388 \\
1\end{array}$ \\
\hline 7 & $\begin{array}{c}5 \\
1.200\end{array}$ & $\begin{array}{c}4.70571 \\
1.129\end{array}$ & $\begin{array}{c}4.56515 \\
1.095\end{array}$ & $\begin{array}{c}4.48283 \\
1.075\end{array}$ & $\begin{array}{c}4.16819 \\
1\end{array}$ \\
\hline 8 & $\begin{array}{c}6 \\
1.167\end{array}$ & $\begin{array}{c}5.69974 \\
1.108\end{array}$ & $\begin{array}{c}5.55530 \\
1.080\end{array}$ & $\begin{array}{c}5.47040 \\
1.0635\end{array}$ & $\begin{array}{c}5.14358 \\
1\end{array}$ \\
\hline 9 & $\begin{array}{c}7 \\
1.143\end{array}$ & $\begin{array}{c}6.695395 \\
1.093\end{array}$ & $\begin{array}{c}6.54810 \\
1.069\end{array}$ & $\begin{array}{c}6.46129 \\
1.055\end{array}$ & $\begin{array}{c}6.12539 \\
1\end{array}$ \\
\hline 10 & $\begin{array}{c}8 \\
1.125\end{array}$ & $\begin{array}{c}7.69208 \\
1.082\end{array}$ & $\begin{array}{c}7.54259 \\
1.061\end{array}$ & $\begin{array}{c}7.45430 \\
1.048\end{array}$ & $\begin{array}{c}.11134 \\
1\end{array}$ \\
\hline
\end{tabular}


2. The crossing of $\mathcal{B}$ at the point $q=2$ for the (infinite-length limit of) strips with global circuits nicely signals the property that the Ising antiferromagnet has a frustrated zero-temperature critical point on these strips. This has been discussed in 40 in the context of exact solutions for finite-temperature Potts model partition functions on the $L_{y}=2$ cyclic and Möbius triangular strips. In contrast, this connection is not, in general, present for strips with free longitudinal boundary conditions since $\mathcal{B}$ does not pass through $q=2$ (as recapitulated below). The $q=3$ Potts antiferromagnet also has a zero-temperature critical point on these strips, and this is similarly manifested by the crossing of the singular locus $\mathcal{B}$ through the point $q=3$ for the cyclic and Möbius strips with $\left(F B C_{y},(T) B C_{x}\right)$, but not for the $L_{y}=3$ torus and Klein bottle strips with $\left(P B C_{y},(T) P B C_{x}\right)$.

3. An interesting feature of the cyclic strips of the triangular lattice (as well as the square and kagomé lattices) is that for all of cases that have been studied here and in [7, 11, 12], there is a correlation between the coefficient $c_{G_{s}, j}$ of the respective dominant $\lambda_{G_{s}, j}$ 's in regions that include intervals of the real axis. Before, it was shown 23] that the $c_{G_{s}, j}$ of the dominant $\lambda_{G_{s}, j}$ in region $R_{1}$ including the real intervals $q>q_{c}(\{G\})$ and $q<0$ is $c^{(0)}=1$, where the $c^{(d)}$ were given in eqs. (2.10), (2.11). Here we extend this, observing that the $c_{G_{s}, j}$ that multiplies the dominant $\lambda_{G_{s}, j}$ in the region containing the intervals $0<q<2$ is $c^{(1)}$. For the cyclic strips of the triangular lattice that we have studied, namely, $L_{y}=2,3,4$, the $c_{G_{s}, j}$ multiplying the dominant $\lambda_{G_{s}, j}$ in the region containing the interval $2<q<3$ is $c^{(2)}$. For the $L_{y}=4$ strip of the triangular lattice, there is another region containing the real interval $3<q<q_{c}$, where $q_{c}$ for this strip was given in (3.7), and we find that $c^{(3)}$ multiplies the dominant $\lambda_{t 4, j}$ containing this interval. For the cyclic strips of the square lattice, although the values of $q_{c}$ are different, a similar correlation is observed; in particular, for the respective widths $L_{y}=3,4, c^{(2)}$ multiplies the $\lambda_{G_{s}, j}$ that is dominant in the region containing the interval $2<q<q_{c}$, where $q_{c} \simeq 2.34$ and $q_{c} \simeq 2.49$ for these two widths 42 .

4. For the strip of the triangular lattice of width $L_{y}=5$ with cylindrical $\left(P B C_{y}, F B C_{x}\right)$ boundary conditions, we find that $\mathcal{B}$ consists of arcs together with a (closed) oval. However, $\mathcal{B}$ does not pass through $q=0,2$, or 3 . This is qualitatively the same morphology that was found for the corresponding strip of width $L_{y}=4$ (see Fig. 4 of [18). For comparison, the triangular strip with width $L_{y}=3$ and $\left(P B C_{y}, F B C_{x}\right)$ had $\mathcal{B}=\emptyset$. The point $q_{c}=4$ for $L_{y}=4$ and $q_{c} \simeq 3.28$ for $L_{y}=5$, which shows that for this family of strips, in cases where there is a $q_{c}$ (there is none for $L_{y}=3$ ), it is not, in general, a nondecreasing function of $L_{y}$. This is somewhat reminiscent of 
the non-monotonicity of $W$ that we showed in the case of $\left(P B C_{y}, B C_{x}\right)$ strips, where $B C_{x}=F B C_{x}$ or $(T) P B C_{x}$, in [20]. For the $L_{y}=6$ strip with $\left(P B C_{y}, F B C_{x}\right)$, we infer from the chromatic zeros that $\mathcal{B}$ consists of a single component, and from the analytic results we compute that $q_{c} \simeq 3.25$, which again shows the nonmonotonicity of $q_{c}$ as a function of $L_{y}$. The morphology of chromatic zeros for our $6 \times 16$ strip is similar to that found in 29] for $8 \times 8$ patch, both with cylindrical boundary conditions. For strips with torus or Klein bottle boundary conditions, $W$ and $\mathcal{B}$ have been calculated for only one $L_{y}$ value, namely, $L_{y}=3$ for the square lattice in [26] and for the triangular lattice here. For the $L_{y}=3\left(P B C_{y},(T) P B C_{x}\right)$ square strip, $q_{c}$ is equal to the value 3 for the full 2D square lattice, but for the $L_{y}=3\left(P B C_{y},(T) P B C_{x}\right)$ triangular strip, our result (4.15) shows that $q_{c}$ is less than the value of 4 for the $2 \mathrm{D}$ triangular lattice.

5. We have included an illustrative result for a wider strip with $\left(F B C_{y}, F B C_{x}\right)$ boundary conditions, namely the strip with $L_{y}=5$. As one increases $L_{y}$, this is the first value at which one can no longer solve analytically for the $\lambda_{j}$ 's as algebraic roots. The locus $\mathcal{B}$ is similar to the respective loci that were found earlier in studies of open strips [17, 18, 19] in that it does not pass through $q=0$ or $q=2$ and does not separate the $q$ plane into regions containing intervals of the real axis. (It does pass through $q=3$, unlike the loci for the open strips with $L_{y}=3,4$.) Our results confirm the trend that was observed in earlier work [17, 18], namely that as $L_{y}$ increases, the arcs elongate and move closer together, and the arc endpoints nearest to the origin move toward this point. In contrast to the strips with global circuits, these strips do not manifest the property that the $q=2$ (Ising) and $q=3$ Potts antiferromagnets have zero-temperature critical points since $\mathcal{B}$ does not pass through these respective points. The simplest example of this is the Ising antiferromagnet on the infinite open line; in this case, although the model has a well-known zero-temperature critical point, this is not evident in the locus $\mathcal{B}$, which is the emptyset.

6. For the strips with $\left(P B C_{y}, F B C_{x}\right)$ and $\left(F B C_{y}, F B C_{x}\right)$, while the endpoints of the arcs on $\mathcal{B}$ that lie closest to the origin tend to move toward the origin as $L_{y}$ increases, leading one to expect that in the limit $L_{y} \rightarrow \infty$, the limiting locus $\mathcal{B}$ would pass through $q=0$, no such motion toward $q=2$ is observed in the cases so far calculated. This is in agreement with the fact that the locus found in [29] for the triangular lattice constructed as the limit $L_{x}, L_{y} \rightarrow \infty$ with $\left(P B C_{y}, F B C_{x}\right)$ boundary conditions passes through $q=0$ and 4 (and at $q \simeq 3$.82) but not through $q=2$ or $q=3$. Thus, assuming that our conjecture $(3.8)$ is correct, it follows that the locus $\mathcal{B}$ for the triangular lattice depends on the boundary conditions used to define this lattice: if one constructs as 
the limit $L_{y} \rightarrow \infty$ with $\left(P B C_{y}, F B C_{x}\right)$ (cylindrical) boundary conditions, then $\mathcal{B}$ does not pass through $q=2$ or 3 [29], while if one constructs it as the limit $L_{y} \rightarrow \infty$ with $\left(F B C_{y},(T) P B C_{x}\right)$ (cyclic or Möbius) boundary conditions, then, if the conjecture is valid, $\mathcal{B}$ would pass through $q=2$ and 3 in the limit just as it does for each of the values $L_{y}=2,3,4$ studied so far. However, since the value of $q_{c}$ pertains directly to a physical quantity, as the minimal value of real $q$ above which $W(q)$ is analytic, one expects that $q_{c}$ should be independent of the boundary conditions used to define the 2D lattice. All results obtained so far are consistent with this expectation.

7. There have been a number of theorems proved concerning real chromatic zeros. An elementary result is that no chromatic zeros can lie on the negative real axis $q<0$, since a chromatic polynomial has alternating coefficients. It has also been proved that there are no chromatic zeros in the intervals $0<q<1$, and $1<q<32 / 27$ [43]. The bound of $32 / 27$ in 43 has been shown to be sharp; i.e., for any $\epsilon>0$, there exists a graph with a chromatic zero at $q=32 / 27+\epsilon$ [44]. See also [45, 34] and references therein. Based on our studies of strips of the triangular (and square) lattices with the various boundary conditions considered, we make the following observation: for such strips, we have not found any chromatic zeros, except for the zero at $q=1$, in the interior of the disk $|q-1|=1$. This motivates the conjecture that for these strips, there are no chromatic zeros with $|q-1|<1$ except for the zero at $q=1$. Assuming that this conjecture is valid, the bound would be a sharp bound, since the circuit graph with $n$ vertices, $C_{n}$, has chromatic zeros lying precisely on the circle $|q-1|=1$ and at $q=1$ [46]. Further work is needed to prove (or disprove) this conjecture.

Some relevant features are summarized in Table 3. The entries for $L_{y}=\infty$ with $\left(P B C_{y}, F B C_{x}\right)$ are from [29].

\section{Conclusions}

In this paper we have presented exact calculations of the zero-temperature $q$-state Potts antiferromagnet partition functions (equivalently, chromatic polynomials $P$ ), on strips of the triangular lattice of width $L_{y}=3$ and with boundary conditions of four types: (a) $\left(F B C_{y}, P B C_{x}\right)=$ cyclic, (b) $\left(F B C_{y}, T P B C_{x}\right)=$ Möbius, (c) $\left(P B C_{y}, P B C_{x}\right)=$ toroidal, and (d) $\left(P B C_{y}, T P B C_{x}\right)=$ Klein bottle, where $F, P$, and $T P$ denote free, periodic, and twisted periodic. In the infinite-length limits of these strips, exact results were given for the ground state degeneracy (exponential of the ground state entropy), $W$, and its analytic structure in the complex $q$ plane, in particular, the nonanalytic locus $\mathcal{B}$, was discussed. Exact calculations 
Table 3: Comparative listing of properties of chromatic polynomials $P$, ground state degeneracy functions $W$, and nonanalytic loci $\mathcal{B}$ for strip graphs $G_{s}$ of the triangular (tri) lattice and their infinite-length limits. New results in this work are marked with asterisks in the first column. The properties apply for a given strip of type $G_{s}$ of size $L_{y} \times L_{x}$; some apply for arbitrary $L_{x}$, such as $N_{\lambda}$, while others apply for the infinite-length limit, such as the properties of the locus $\mathcal{B}$. For the boundary conditions in the $y$ and $x$ directions $\left(B C_{y}, B C_{x}\right), \mathrm{F}, \mathrm{P}$, and $\mathrm{T}$ denote free, periodic, and orientation-reversed (twisted) periodic, and the notation $(\mathrm{T}) \mathrm{P}$ means that the results apply for either periodic or orientation-reversed periodic. The column denoted eqs. describes the numbers and degrees of the algebraic equations giving the $\lambda_{G_{s}, j}$ in $P$; for example, $\{3(1), 2(2), 1(3)\}$ indicates that there are 3 linear equations, 2 quadratic equations and one cubic equation. The column denoted BCR lists the points at which $\mathcal{B}$ crosses the real $q$ axis; the largest of these is $q_{c}$ for the given family $G_{s}$. The notation "none" in this column indicates that $\mathcal{B}$ does not cross the real $q$ axis. Column labelled "SN" refers to whether $\mathcal{B}$ has support for negative $\operatorname{Re}(q)$, indicated as yes (y) or no (n).

\begin{tabular}{|c|c|c|c|c|c|c|c|}
\hline \hline$G_{s}$ & $L_{y}$ & $B C_{y}$ & $B C_{x}$ & $N_{\lambda}$ & eqs. & BCR & SN \\
\hline \hline tri & 2 & $\mathrm{~F}$ & $(\mathrm{~T}) \mathrm{P}$ & 4 & $\{2(1), 1(2)\}$ & $3,2,0$ & $\mathrm{n}$ \\
\hline$* * \operatorname{tri}$ & 3 & $\mathrm{~F}$ & $(\mathrm{~T}) \mathrm{P}$ & 10 & $\{3(1), 2(2), 1(3)\}$ & $3,2,0$ & $\mathrm{n}$ \\
\hline$* * \operatorname{tri}$ & 4 & $\mathrm{~F}$ & $\mathrm{P}$ & 26 & $\{1(1), 2(4), 1(8), 1(9)\}$ & $3.23,3,2,0$ & $\mathrm{y}$ \\
\hline \hline tri & 3 & $\mathrm{P}$ & $\mathrm{F}$ & 1 & $\{1(1)\}$ & none & - \\
\hline tri & 4 & $\mathrm{P}$ & $\mathrm{F}$ & 2 & $\{1(2)\}$ & $4,3.48$ & $\mathrm{n}$ \\
\hline$* * \operatorname{tri}$ & 5 & $\mathrm{P}$ & $\mathrm{F}$ & 2 & $\{1(2)\}$ & $3.28,3.21$ & $\mathrm{n}$ \\
\hline$* * \operatorname{tri}$ & 6 & $\mathrm{P}$ & $\mathrm{F}$ & 5 & $\{1(5)\}$ & 3.25 & $\mathrm{n}$ \\
\hline tri & $\infty$ & $\mathrm{P}$ & $\mathrm{F}$ & - & - & $4,3.82,0$ & $\mathrm{y}$ \\
\hline \hline$* * \operatorname{tri}$ & 3 & $\mathrm{P}$ & $\mathrm{P}$ & 11 & $\{5(1), 3(2)\}$ & $3.72,2,0$ & $\mathrm{n}$ \\
\hline$* * \operatorname{tri}$ & 3 & $\mathrm{P}$ & $\mathrm{TP}$ & 5 & $\{5(1)\}$ & $3.72,2,0$ & $\mathrm{n}$ \\
\hline \hline tri & 2 & $\mathrm{~F}$ & $\mathrm{~F}$ & 1 & $\{1(1)\}$ & none & - \\
\hline tri & 3 & $\mathrm{~F}$ & $\mathrm{~F}$ & 2 & $\{1(2)\}$ & 2.57 & $\mathrm{n}$ \\
\hline tri & 4 & $\mathrm{~F}$ & $\mathrm{~F}$ & 4 & $\{1(4)\}$ & none & $\mathrm{n}$ \\
\hline$* * \operatorname{tri}$ & 5 & $\mathrm{~F}$ & $\mathrm{~F}$ & 9 & $\{1(9)\}$ & 3 & $\mathrm{n}$ \\
\hline \hline
\end{tabular}


of $P$ and $W$ and studies of $\mathcal{B}$ were also presented for wider strips, including (e) cyclic, $L_{y}=4$, (f) $\left(P B C_{y}, F B C_{x}\right)=$ cylindrical, $L_{y}=5,6$, and an illustrative $\left(F B C_{y}, F B C_{x}\right)=$ open case with $L_{y}=5$. A comparative analysis of these results was included. An interesting result of our calculations of $W$ on infinite-length strips with different widths and transverse boundary conditions is the observation that for the cases studied, $\mathcal{B}$ passes through $q=2$ (as well as $q=0$ ) for strips with periodic or twisted periodic longitudinal boundary conditions but does not for strips with free longitudinal boundary conditions. Hence, in particular, if one uses periodic or twisted periodic longitudinal boundary conditions, the locus $\mathcal{B}$ nicely signals the existence of the zero-temperature critical point of the Ising antiferromagnet on these infinitelength, finite-width strips of the triangular lattice. Numerical values of $W$ were given for infinite-length strips of various widths and were shown to approach values for the 2D lattice rapidly. Some conjectures for the behavior of $\mathcal{B}$ for arbitrarily wide strips, and for a region in the $q$ plane free of chromatic zeros, were stated. These exact calculations of the $T=0$ Potts

antiferromagnet partition function and ground state degeneracy on strips of the triangular lattice give valuable analytic knowledge of properties of Potts antiferromagnets.

Note added: The original version of this paper, submitted in early Oct. 1999, contained calculations on $L_{y}=3$ strips of the triangular lattice. In response to a request by a referee to perform calculations for wider strips, we have added the results on the $L_{y}=4$ strip with $\left(F B C_{y}, P B C_{x}\right)$, the $L_{y}=5,6$ strips with $\left(P B C_{y}, F B C_{x}\right)$, and the $L_{y}=5$ strip with $\left(F B C_{y}, F B C_{x}\right)$.

Acknowledgment: The research of R. S. was supported in part by the U. S. NSF grant PHY-97-22101.

\section{Appendix}

\subsection{Generating Function for $L_{y}=3$ Möbius Strip of the Triangular Lattice}

As noted in the text, it is convenient to leave the coefficients $c_{t 3 M b, j}, j=6,7,8$ in the general form (2.37). For the evaluation of these coefficients, we list here the generating function for 
this strip. We have $d_{\mathcal{N}}=8$ and $d_{\mathcal{D}}=10$, and

$$
\mathcal{D}\left(\operatorname{tri}\left(L_{y}=3\right), F B C_{y}, T P B C_{x}, q, x\right)=\prod_{j=1}^{10}\left(1-\lambda_{t 3, j}(q) x\right)
$$

where the $\lambda_{t 3, j}$ were given in eq. (2.15). Since several of the $\lambda_{t 3, j}$ 's are algebraic, it is useful to display the denominator in an explicitly polynomial form:

$$
\begin{aligned}
& \mathcal{D}\left(\operatorname{tri}\left(L_{y}=3\right), F B C_{y}, T P B C_{x}, q, x\right)=(1+x)[1-(q-2) x] \times \\
& {\left[1-(2 q-7) x+(q-2)(q-3) x^{2}\right][1+(q-2)(q-3) x] F_{t 3 q 3} F_{t 3 q 2}}
\end{aligned}
$$

where

$$
F_{t 3 q 3}=\prod_{j=6,7,8}\left(1-\lambda_{t 3, j} x\right)=1+b_{t 3,1} x+b_{t 3,2} x^{2}+b_{t 3,3} x^{3}
$$

with $b_{t 3, j}, j=1,2,3$ given in eqs. (2.18)-(2.20), and

$$
F_{t 3 q 2}=\prod_{j=9,10}\left(1-\lambda_{t, j} x\right)=1-\left(q^{3}-7 q^{2}+18 q-17\right) x+(q-2)^{3}(q-3) x^{2} .
$$

For the numerator, extracting a common factor via the definition of the reduced coefficients $\bar{A}_{t 3 M b, j}$,

$$
A_{t 3 M b, j} \equiv q(q-1)(q-2)(q-3) \bar{A}_{t 3 M b, j}
$$

we have

$$
\begin{gathered}
\bar{A}_{t 3 M b, 0}=q^{2}-6 q+10 \\
\bar{A}_{t 3 M b, 1}=(q-3)\left(q^{3}-12 q^{2}+45 q-55\right) \\
\bar{A}_{t 3 M b, 2}=q^{6}-20 q^{5}+170 q^{4}-779 q^{3}+2016 q^{2}-2779 q+1588 \\
\bar{A}_{t 3 M b, 3}=-(q-3)\left(5 q^{6}-82 q^{5}+574 q^{4}-2185 q^{3}+4745 q^{2}-5536 q+2691\right) \\
\bar{A}_{t 3 M b, 4}=(q-2)(q-3)\left(9 q^{6}-141 q^{5}+930 q^{4}-3303 q^{3}+6651 q^{2}-7176 q+3224\right) \\
\bar{A}_{t 3 M b, 5}=-(q-2)^{2}(q-3)^{3}\left(6 q^{4}-55 q^{3}+186 q^{2}-277 q+152\right) \\
\bar{A}_{t 3 M b, 6}=-(q-2)^{5}(q-3)\left(q^{4}-12 q^{3}+59 q^{2}-138 q+125\right) \\
\bar{A}_{t 3 M b, 7}=(q-2)^{6}(q-3)^{3}\left(3 q^{2}-14 q+17\right) \\
\bar{A}_{t 3 M b, 8}=-(q-2)^{9}(q-3)^{3} .
\end{gathered}
$$

It should also be noted that there is a significant difference between the strips of the triangular lattice studied here and the analogous strips of the square lattice [11, 12, 26]. In 
general, a strip of the square lattice of width $L_{y}$ and length $L_{x}$ with any of the boundary conditions $\left(F B C_{y}, P B C_{x}\right)$ (cyclic), ( $\left.F B C_{y}, T P B C_{x}\right)$ (Möbius), $\left(P B C_{y}, P B C_{x}\right)$ (torus), or $\left(P B C_{y}, T P B C_{x}\right)$ (Klein bottle) is invariant under a translation by one edge or vertex in the longitudinal direction. However, in the case of the analogous strip of the triangular lattice, this is only true of the cases with cyclic and torus boundary conditions; the strips with Möbius and Klein bottle boundary conditions have a "seam" along with the orientation of the triangles reverses. This is discussed further in the appendix. Thus, if one proceeds in a longitudinal direction along the triangular-lattice Möbius strip, starting in a manner such that the triangles are formed by edges connecting the upper left and lower right vertices of squares (relative to one's direction of motion), then when one crosses this seam, the triangles will be formed by edges connecting the upper right and lower left vertices of the squares on the strip. Related to this, there are differences in the degrees of various vertices on these strips (where the degree of a vertex is defined as the number of edges that connect to this vertex). If one avoids the lowest few values of $L_{x}$ where the strips degenerate, then, in general, (i) for the cyclic triangular strip of width $L_{y}$ and length $L_{x}$, the $\left(L_{y}-2\right) L_{x}$ internal vertices have degree 6 while the $2 L_{x}$ vertices on the upper and lower sides have degree 4 ; (ii) for the same strip as in (i) but with Möbius instead of cyclic longitudinal boundary conditions, the $\left(L_{y}-2\right) L_{x}$ internal vertices have degree 6 , the $2\left(L_{y}-1\right)$ vertices on the upper and lower sides except for those on the seam have degree 4 , and, on the seam, the external vertices have degrees 5 and 3; (iii) for the same strip as in (i) but with torus or Klein bottle boundary conditions, all of the vertices have degree 6 .

\subsection{Equations for the Terms in the Chromatic Polynomial for the Cyclic $L_{y}=4$ Strip of the Triangular Lattice}

Four of the $\lambda_{t 4, j}$, which we label as $j=2,3,4,5$, are the same as for the open $L_{y}=4$ strip of the triangular lattice. These are solutions to the quartic equation

$$
\xi^{4}+b_{t 4,1,1} \xi^{3}+b_{t 4,1,2} \xi^{2}+b_{t 4,1,3} \xi+b_{t 4,1,4}=0
$$

where the coefficients were given as $b_{t(4), j} \equiv b_{t 4,1, j}, j=1, . ., 4$ in eqs. (B.15)-(B.18) of [17]

The terms $\lambda_{t 4, j}, j=6,7,8,9$ are solutions to the quartic equation

$$
\xi^{4}+b_{t 4,2,1} \xi^{3}+b_{t 4,2,2} \xi^{2}+b_{t 4,2,3} \xi+b_{t 4,2,4}=0
$$

where

$$
\begin{gathered}
b_{t 4,2,1}=4 q-13 \\
b_{t 4,2,2}=2\left(3 q^{2}-18 q+26\right)
\end{gathered}
$$




$$
b_{t 4,2,3}=(q-2)\left(4 q^{2}-25 q+38\right)
$$

and

$$
b_{t 4,2,4}=(q-2)^{2}(q-3)^{2} .
$$

Another set of $\lambda_{j}$ 's for $10 \leq j \leq 17$ are roots of an equation of degree 8 ,

$$
\xi^{8}+\sum_{k=1}^{8} b_{t 4,3, k} \xi^{8-k}=0
$$

where

$$
\begin{array}{cr}
b_{t 4,3,1}=2\left(-3 q^{2}+19 q-31\right) & (9.2 .8) \\
b_{t 4,3,2}=15 q^{4}-186 q^{3}+867 q^{2}-1794 q+1385 & (9.2 .9) \\
b_{t 4,3,3}=-20 q^{6}+366 q^{5}-2784 q^{4}+11248 q^{3}-25425 q^{2}+30452 q-15080 & (9.2 .10) \\
b_{t 4,3,4}=(q-2)\left(15 q^{7}-334 q^{6}+3174 q^{5}-16676 q^{4}+52294 q^{3}-97852 q^{2}+101138 q-44528\right) \\
b_{t 4,3,5}=-(q-2)^{2}(q-3)^{2}\left(6 q^{6}-126 q^{5}+1076 q^{4}-4804 q^{3}+11861 q^{2}-15378 q+8185\right) & (9.2 .12) \\
b_{t 4,3,6}=(q-2)^{4}(q-3)^{3}\left(q^{5}-25 q^{4}+216 q^{3}-868 q^{2}+1670 q-1246\right) & (9.2 .13) \\
b_{t 4,3,7}=(q-2)^{6}(q-3)^{4}\left(2 q^{3}-22 q^{2}+80 q-97\right) & (9.2 .14) \\
b_{t 4,3,8}=(q-2)^{8}(q-3)^{6} . & (9.2 .15)
\end{array}
$$

A final set of $\lambda_{t 4, j}, 18 \leq j \leq 26$, are solutions to the equation of degree 9

$$
\xi^{9}+\sum_{k=1}^{9} b_{t 4,4, k} \xi^{9-k}=0
$$

where

$$
\begin{gathered}
b_{t 4,4,1}=2(q-3)\left(2 q^{2}-12 q+21\right) \\
b_{t 4,4,2}=(q-3)\left(6 q^{5}-90 q^{4}+558 q^{3}-1772 q^{2}+2865 q-1875\right) \\
b_{t 4,4,3}=4 q^{9}-111 q^{8}+1380 q^{7}-10071 q^{6}+47476 q^{5}-149742 q^{4}+315652 q^{3} \\
-428385 q^{2}+339300 q-119368 \\
b_{t 4,4,4}=(q-2)(q-3)^{2}\left(q^{9}-34 q^{8}+491 q^{7}-4032 q^{6}+20961 q^{5}-71954 q^{4}\right. \\
\left.+163654 q^{3}-238278 q^{2}+201722 q-75672\right)
\end{gathered}
$$




$$
\begin{gathered}
b_{t 4,4,5}=-(q-2)^{2}(q-3)^{3}\left(3 q^{9}-81 q^{8}+983 q^{7}-7029 q^{6}+32609 q^{5}-101701 q^{4}\right. \\
\left.+213036 q^{3}-288702 q^{2}+229385 q-81299\right) \\
b_{t 4,4,6}=(q-2)^{4}(q-3)^{4}\left(3 q^{8}-70 q^{7}+728 q^{6}-4404 q^{5}+16929 q^{4}-42286 q^{3}\right. \\
\left.\quad+66933 q^{2}-61296 q+24830\right) \\
b_{t 4,4,7}=-(q-2)^{6}(q-3)^{6}\left(q^{6}-20 q^{5}+166 q^{4}-734 q^{3}+1833 q^{2}-2462 q+1393\right) \\
b_{t 4,4,8}=-(q-2)^{8}(q-3)^{7}\left(2 q^{4}-21 q^{3}+87 q^{2}-165 q+119\right) \\
b_{t 4,4,9}=-(q-2)^{12}(q-3)^{8} .
\end{gathered}
$$

\subsection{Generating Function for the $L_{y}=5,6$ Strips of the Triangular Lattice with $\left(P B C_{y}, F B C_{x}\right)$}

For the $L_{y}=5$ strip we calculate a generating function of the form (2.2) with $d_{\mathcal{D}}=2, d_{\mathcal{N}}=1$ and, in the notation of eqs. (2.3) and (2.4),

$$
b_{t 5 P F, 1}=-q^{5}+15 q^{4}-98 q^{3}+355 q^{2}-711 q+614
$$

$$
b_{t 5 P F, 2}=(q-3)\left(3 q^{7}-66 q^{6}+619 q^{5}-3205 q^{4}+9877 q^{3}-18065 q^{2}+18078 q-7588\right)
$$

$$
A_{t 5 P F, 0}=q(q-1)(q-2)(q-3)\left(q^{6}-14 q^{5}+85 q^{4}-290 q^{3}+599 q^{2}-723 q+398\right)
$$

$$
\begin{aligned}
& A_{t 5 P F, 1}=-q(q-1)(q-2)(q-3)\left(q^{2}-2 q+2\right)\left(3 q^{7}-66 q^{6}+619 q^{5}-3205 q^{4}\right. \\
& \left.+9877 q^{3}-18065 q^{2}+18078 q-7588\right)
\end{aligned}
$$

For the $L_{y}=6$ strip we calculate a generating function of the form $(2.2)$ with $d_{\mathcal{D}}=5$, $d_{\mathcal{N}}=4$ and

$$
b_{t 6 P F, 1}=-q^{6}+18 q^{5}-145 q^{4}+680 q^{3}-1980 q^{2}+3379 q-2586
$$




$$
\begin{aligned}
& b_{t 6 P F, 2}=4 q^{10}-128 q^{9}+1868 q^{8}-16352 q^{7}+94977 q^{6}-382031 q^{5}+1076317 q^{4} \\
& -2093899 q^{3}+2686606 q^{2}-2047842 q+702080
\end{aligned}
$$

$$
\begin{aligned}
& b_{t 6 P F, 3}=-2(q-3)^{2}\left(q^{12}-39 q^{11}+695 q^{10}-7493 q^{9}+54509 q^{8}-282283 q^{7}+1068575 q^{6}\right. \\
& \left.-2982861 q^{5}+6098756 q^{4}-8908956 q^{3}+8820488 q^{2}-5306146 q+1462992\right)
\end{aligned}
$$

$$
\begin{aligned}
& b_{t 6 P F, 4}=-2(q-2)(q-3)^{5}\left(2 q^{11}-62 q^{10}+882 q^{9}-7601 q^{8}+44105 q^{7}-181018 q^{6}\right. \\
& \left.+536536 q^{5}-1149015 q^{4}+1742334 q^{3}-1779827 q^{2}+1099188 q-309188\right) \\
& b_{t 6 P F, 5}=4(q-2)^{2}(q-3)^{8}\left(q^{2}-5 q+5\right)\left(2 q^{7}-36 q^{6}+277 q^{5}-1179 q^{4}+2990 q^{3}\right. \\
& \left.-4505 q^{2}+3728 q-1310\right)
\end{aligned}
$$

$$
\begin{aligned}
& A_{t 6 P F, 0}=q(q-1)(q-2)\left(q^{9}-21 q^{8}+199 q^{7}-1121 q^{6}+4159 q^{5}-10623 q^{4}\right. \\
& \left.+18887 q^{3}-22824 q^{2}+17177 q-6143\right)
\end{aligned}
$$

$$
\begin{aligned}
& A_{t 6 P F, 1}=-q(q-1)(q-2)\left(4 q^{13}-140 q^{12}+2266 q^{11}-22416 q^{10}+150973 q^{9}\right. \\
& -730186 q^{8}+2607252 q^{7}-6958852 q^{6}+13899608 q^{5}-20584349 q^{4} \\
& \left.+22103679 q^{3}-16461349 q^{2}+7723994 q-1748140\right)
\end{aligned}
$$

$$
A_{t 6 P F, 2}=2 q(q-1)(q-2)(q-3)^{2}\left(q^{15}-42 q^{14}+816 q^{13}-9734 q^{12}+79793 q^{11}\right.
$$




$$
\begin{aligned}
& -476549 q^{10}+2144264 q^{9}-7409966 q^{8}+19852299 q^{7}-41297346 q^{6}+66301130 q^{5} \\
& \left.-80939629 q^{4}+73099740 q^{3}-46448750 q^{2}+18742947 q-3655548\right) \\
& A_{t 6 P F, 3}=2 q(q-1)(q-2)(q-3)^{5}\left(2 q^{15}-72 q^{14}+1212 q^{13}-12651 q^{12}+91556 q^{11}\right. \\
& -486599 q^{10}+1962326 q^{9}-6116898 q^{8}+14870220 q^{7}-28223745 q^{6}+41554711 q^{5} \\
& \left.-46735427 q^{4}+39026466 q^{3}-22975509 q^{2}+8586616 q-1545752\right) \\
& A_{t 6 P F, 4}=-4 q(q-1)(q-2)^{2}(q-3)^{8}\left(q^{2}-5 q+5\right)\left(q^{4}-5 q^{3}+10 q^{2}-10 q+5\right) \\
& \times\left(2 q^{7}-36 q^{6}+277 q^{5}-1179 q^{4}+2990 q^{3}-4505 q^{2}+3728 q-1310\right)
\end{aligned}
$$

\subsection{Generating Function for $L_{y}=5$ Open Strip of the Triangular Lattice}

For this strip we calculate a generating function of the form (2.2) with $d_{\mathcal{D}}=9, d_{\mathcal{N}}=8$ and, in the notation of eqs. (2.3) and (2.4),

$$
\begin{gathered}
b_{t 5 F F, 1}=-(q-3)\left(q^{4}-10 q^{3}+46 q^{2}-113 q+120\right) \\
b_{t 5 F F, 2}=6 q^{8}-131 q^{7}+1280 q^{6}-7328 q^{5}+26930 q^{4}-65081 q^{3}+100888 q^{2}-91462 q+36965 \\
b_{t 5 F F, 3}=-(q-2)(q-3)\left(15 q^{9}-378 q^{8}+4289 q^{7}-28788 q^{6}+126096 q^{5}-374139 q^{4}\right. \\
\left.+752541 q^{3}-989867 q^{2}+772611 q-272483\right) \\
b_{t 5 F F, 4}=(q-2)(q-3)^{2}\left(20 q^{11}-607 q^{10}+8429 q^{9}-70702 q^{8}+398115 q^{7}\right.
\end{gathered}
$$




$$
\begin{aligned}
& -1580547 q^{6}+4515585 q^{5}-9285872 q^{4}+13471537 q^{3}-13131321 q^{2} \\
& +7738560 q-2087938)
\end{aligned}
$$

$$
\begin{aligned}
& b_{t 5 F F, 5}=-(q-2)^{2}(q-3)^{3}\left(15 q^{12}-502 q^{11}+7729 q^{10}-72397 q^{9}\right. \\
& +459566 q^{8}-2083176 q^{7}+6915864 q^{6}-16947196 q^{5}+30430188 q^{4} \\
& \left.-39053679 q^{3}+34008163 q^{2}-18041392 q+4408580\right)
\end{aligned}
$$

$$
\begin{aligned}
& b_{t 5 F F, 6}=(q-2)^{3}(q-3)^{5}\left(6 q^{12}-203 q^{11}+3149 q^{10}-29630 q^{9}+188440 q^{8}-853749 q^{7}\right. \\
& +2826657 q^{6}-6893466 q^{5}+12293272 q^{4}-15636918 q^{3}+13466958 q^{2} \\
& -7049578 q+1695556)
\end{aligned}
$$

$$
\begin{aligned}
& b_{t 5 F F, 7}=-(q-2)^{5}(q-3)^{7}\left(q^{11}-34 q^{10}+516 q^{9}-4647 q^{8}+27718 q^{7}-115308 q^{6}\right. \\
& \left.+342008 q^{5}-724072 q^{4}+1072964 q^{3}-1060043 q^{2}+628196 q-169014\right) \\
& b_{t 5 F F, 8}=-(q-2)^{7}(q-3)^{9}\left(q^{9}-23 q^{8}+241 q^{7}-1505 q^{6}+6145 q^{5}-16929 q^{4}\right. \\
& \left.+31319 q^{3}-37359 q^{2}+25972 q-7987\right)
\end{aligned}
$$

$$
b_{t 5 F F, 9}=(q-2)^{12}(q-3)^{11}\left(q^{3}-8 q^{2}+21 q-17\right)
$$

We use the definition $A_{t 5 F F, j}=q(q-1)(q-2) \bar{A}_{t 5 F F, j}$ and have

$$
\bar{A}_{t 5 F F, 0}=(q-2)^{7}
$$




$$
\begin{aligned}
& \bar{A}_{t 5 F F, 1}=-(q-2)^{2}\left(6 q^{8}-113 q^{7}+926 q^{6}-4304 q^{5}+12381 q^{4}-22504 q^{3}\right. \\
& \left.+25133 q^{2}-15663 q+4121\right)
\end{aligned}
$$

$$
\begin{aligned}
& \bar{A}_{t 5 F F, 2}=(q-2)^{2}(q-3)\left(15 q^{10}-363 q^{9}+3936 q^{8}-25141 q^{7}+104572 q^{6}\right. \\
& \left.-295342 q^{5}+572184 q^{4}-748554 q^{3}+630336 q^{2}-306798 q+65006\right)
\end{aligned}
$$

$$
\begin{aligned}
& \bar{A}_{t 5 F F, 3}=-(q-2)^{2}(q-3)^{2}\left(20 q^{12}-587 q^{11}+7867 q^{10}-63587 q^{9}+344801 q^{8}\right. \\
& -1319670 q^{7}+3650087 q^{6}-7338694 q^{5}+10623070 q^{4}-10770336 q^{3}+7237158 q^{2} \\
& -2882235 q+511741)
\end{aligned}
$$

$$
\begin{aligned}
& \bar{A}_{t 5 F F, 4}=(q-2)^{3}(q-3)^{3}\left(15 q^{13}-487 q^{12}+7266 q^{11}-65902 q^{10}+405008 q^{9}\right. \\
& -1779077 q^{8}+5739974 q^{7}-13753341 q^{6}+24433426 q^{5}-31724357 q^{4}+29191727 q^{3} \\
& \left.-17973781 q^{2}+6613085 q-1093258\right)
\end{aligned}
$$

$$
\begin{aligned}
& \bar{A}_{t 5 F F, 5}=-(q-2)^{4}(q-3)^{4}\left(6 q^{14}-215 q^{13}+3554 q^{12}-35907 q^{11}+247594 q^{10}\right. \\
& -1231849 q^{9}+4556733 q^{8}-12718548 q^{7}+26883798 q^{6}-42760478 q^{5}+50287310 q^{4} \\
& \left.-42307379 q^{3}+24007733 q^{2}-8200007 q+1267630\right)
\end{aligned}
$$

$$
\bar{A}_{t 5 F F, 6}=(q-2)^{6}(q-3)^{7}\left(q^{12}-33 q^{11}+485 q^{10}-4225 q^{9}+24378 q^{8}-98266 q^{7}\right.
$$




$$
\begin{gathered}
\left.+283651 q^{6}-589907 q^{5}+875195 q^{4}-900611 q^{3}+607987 q^{2}-240848 q+42203\right) \\
\bar{A}_{t 5 F F, 7}=(q-2)^{10}(q-3)^{9}\left(q^{8}-18 q^{7}+145 q^{6}-674 q^{5}+1941 q^{4}-3474 q^{3}\right. \\
\left.+3701 q^{2}-2120 q+499\right) \\
\bar{A}_{t 5 F F, 8}=-(q-1)^{3}(q-2)^{11}(q-3)^{11}\left(q^{3}-8 q^{2}+21 q-17\right)
\end{gathered}
$$

\section{5 $L_{y}=2$ Cyclic and Möbius Strips of the Triangular Lattice with $\operatorname{Odd} N_{t}$}

In this part of the appendix we shall report some new results for $L_{y}=2$ cyclic and Möbius strips of the triangular lattice with an odd number $N_{t}$ of triangles and compare these with the case of even $N_{t}=L_{x} L_{y}$. Some illustrative strip graphs are shown in Fig. 8 .

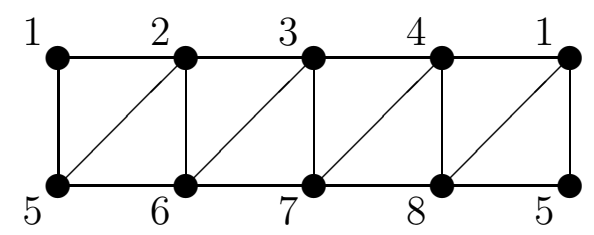

(a)

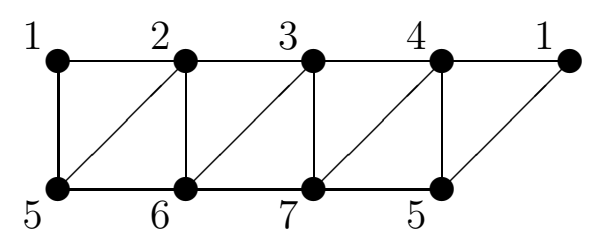

(c)

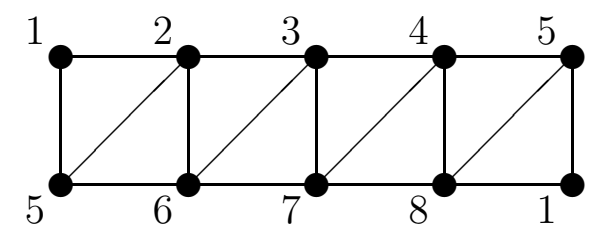

(b)

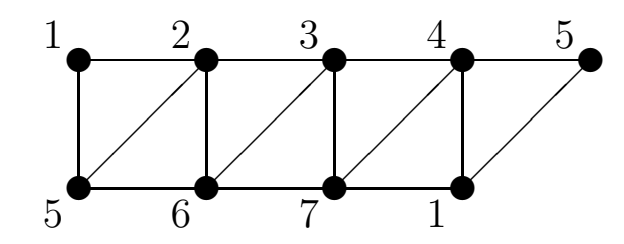

(d)

Figure 8: Illustrative strip graphs of the triangular lattice with width $L_{y}=2$ : (a) cyclic, $L_{x}=4$, even $N_{t}=2 L_{x} ;$ (b) Möbius, $L_{x}=4$, even $N_{t}=2 L_{x}$; (c) cyclic, odd $N_{t}=7 ;$ (d) Möbius, odd $N_{t}=7$.

For the $L_{y}=2$ cyclic strip of the triangular lattice with even $N_{t}=2 L_{x}$ we calculated 
11] (see also [30, 27])

$$
P\left(\operatorname{tri}\left(L_{y}=2, \text { cyc. }\right), N_{t}=2 m, q\right)=\left(q^{2}-3 q+1\right)+\left[(q-2)^{2}\right]^{m}+(q-1)\left[\left(\lambda_{t 2,3}\right)^{m}+\left(\lambda_{t 2,4}\right)^{m}\right]
$$

where

$$
\lambda_{t 2,(3,4)}=\frac{1}{2}[5-2 q \pm \sqrt{9-4 q}] .
$$

For the corresponding $L_{y}=2$ Möbius strip of the triangular lattice with even $N_{t}=2 L_{x}$, we calculated [1]

$$
P\left(\operatorname{tri}\left(L_{y}=2, M b .\right), N_{t}=2 m, q\right)=-1+\left[(q-2)^{2}\right]^{m}-(q-1)(q-3) \frac{\left[\left(\lambda_{t 2,3}\right)^{m}-\left(\lambda_{t 2,4}\right)^{m}\right]}{\lambda_{t 2,3}-\lambda_{t 2,4}} .
$$

Note that the Möbius strip has a seam. The function $W$ and the boundary $\mathcal{B}$ were given in [11]; $\mathcal{B}$ separates the $q$ plane into three regions and crosses the real axis at $q=0,2$, and at $q_{c}=3$.

We proceed to our new results. For the $L_{y}=2$ cyclic strip of the triangular lattice containing an odd number $N_{t}=2 m+1$ of triangles, we calculate

$$
\begin{aligned}
& P\left(\operatorname{tri}\left(L_{y}=2, \text { cyc. }\right), N_{t}=2 m+1, q\right)=-\left(q^{2}-3 q+1\right)+(q-2)\left[(q-2)^{2}\right]^{m}+ \\
& \frac{1}{2}(q-1)(q-3)\left[\left(\left(\lambda_{t 2,3}\right)^{m}+\left(\lambda_{t 2,4}\right)^{m}\right)+\frac{\left(\left(\lambda_{t 2,3}\right)^{m}-\left(\lambda_{t 2,4}\right)^{m}\right)}{\lambda_{t 2,3}-\lambda_{t 2,4}}\right]
\end{aligned}
$$

This strip has a seam.

For the $L_{y}=2$ Möbius strip of the triangular lattice with an odd number $N_{t}=2 m+1$ of triangles (which does not have a seam) we obtain

$$
\begin{aligned}
& P\left(\operatorname{tri}\left(L_{y}=2, M b .\right), N_{t}=2 m+1, q\right)=1+(q-2)\left[(q-2)^{2}\right]^{m}+ \\
& \frac{1}{2}(1-q)\left[\left(\left(\lambda_{t 2,3}\right)^{m}+\left(\lambda_{t 2,4}\right)^{m}\right)+(9-4 q) \frac{\left(\left(\lambda_{t 2,3}\right)^{m}-\left(\lambda_{t 2,4}\right)^{m}\right)}{\lambda_{t 2,3}-\lambda_{t 2,4}}\right] .
\end{aligned}
$$

Note that the sum of the coefficients $C=0$ for the chromatic polynomials for both of the $L_{y}=2$ strips with odd $N_{t}$, eqs. (9.5.4) and (9.5.5). For comparison, for the $L_{y}=2$ even- $N_{t}$ strips, $C=q(q-1)$ for the cyclic case, eq. (9.5.1) and $C=0$ for the Möbius case, eq. (9.5.3).

Equivalently, one may write these results in terms of generating functions, with the definition analogous to (2.1):

$$
\Gamma\left(\operatorname{tri}\left(L_{y}=2, B C_{x}\right), N_{t}=\text { even }, q, x\right)=\sum_{m=2}^{\infty} P\left(\operatorname{tri}\left(L_{y}=2, B C_{x}\right), N_{t}=2 m, q\right) x^{m-2} .
$$


and

$$
\Gamma\left(\operatorname{tri}\left(L_{y}=2, B C_{x}\right), N_{t}=o d d, q, x\right)=\sum_{m=2}^{\infty} P\left(\operatorname{tri}\left(L_{y}=2, B C_{x}\right), N_{t}=2 m+1, q\right) x^{m-2}
$$

where $B C_{x}=P B C_{x}$ or $T P B C_{x}$. The generating functions for all of these strips have the same denominator,

$$
\begin{aligned}
& \mathcal{D}\left(\operatorname{tri}\left(L_{y}=2, \text { cyc. }\right)\right)=\prod_{j=1}^{4}\left(1-\lambda_{t 2, j} x\right) \\
& =(1-x)\left[1-(q-2)^{2} x\right]\left[1-(5-2 q) x+(q-2)^{2} x^{2}\right] .
\end{aligned}
$$

The numerators are easily worked out from the results that we have given for the chromatic polynomials and the denominator $\mathcal{D}$; for example,

$$
\begin{gathered}
\Gamma\left(\operatorname{tri}\left(L_{y}=2, \text { cyc. }\right), N_{t}=\text { even, } q, x\right)=\frac{q(q-1)(q-2)\left[q-3+(q-2) x-(q-2)^{3} x^{2}\right]}{\mathcal{D}\left(\operatorname{tri}\left(L_{y}=2, c y c .\right)\right)} \\
\Gamma\left(\operatorname{tri}\left(L_{y}=2, \text { cyc. }\right), N_{t}=o d d, q, x\right)=\frac{q(q-1)(q-2)(q-3)\left[q-3+(q-2)^{2} x\right]}{\mathcal{D}\left(\operatorname{tri}\left(L_{y}=2, c y c .\right)\right)}
\end{gathered}
$$

and so forth for the Möbius strips.

We remark on some general features of these results. The chromatic polynomials for both even and odd $N_{t}$ and both cyclic and Möbius strips have the same four $\lambda_{j}$ 's, and hence the same $W$ functions and boundary $\mathcal{B}$ (given in [11]). For all cases, $q_{c}=3$. These properties are

in agreement with the general discussion in [11, 12, 23] on the effects of boundary conditions on $W$ and $\mathcal{B}$.

\section{References}

[1] Potts, R. B. 1952 Proc. Camb. Phil. Soc. 48, 106.

[2] Wu, F. Y. 1982 Rev. Mod. Phys. 54, 235.

[3] Read, R. C. 1968 J. Combin. Theory 4, 52.

[4] Tutte, W. T. 1974 "Chromials", in Lecture Notes in Math. v. 411, p. 243.

[5] Read, R. C. and Tutte, W. T. 1988 "Chromatic Polynomials", in Selected Topics in Graph Theory, 3, (Academic Press, New York), p. 15. 
[6] Biggs, N. L. 1993 Algebraic Graph Theory (Cambridge Univ. Press, Cambridge).

[7] Shrock, R. and Tsai, S.-H. 1997 Phys. Rev. E55, 5165.

[8] Biggs, N. L., Damerell, R. M. and Sands, D. A. 1972 J. Combin. Theory B 12, 123.

[9] Biggs, N. L. and Meredith, G. H. 1976 J. Combin. Theory B 20, 5.

[10] Biggs, N. L. 1977 Bull. London Math. Soc. 9, 54.

[11] Shrock, R. and Tsai, S.-H. 1999 Phys. Rev. E60, 3512; Physica A 275, 429.

[12] Shrock, R. 1999 Phys. Lett. A261, 57.

[13] Beraha, S. and Kahane, J. 1979 J. Combin. Theory B 27, 1.

[14] Beraha, S., Kahane, J., and Weiss, N. 1980 J. Combin. Theory B 28, 52.

[15] Read, R. C. 1988 in Proc. 5th Caribbean Conf. on Combin. and Computing.

[16] Read, R. C. and Royle, G. F. 1991 in Graph Theory, Combinatorics, and Applications (Wiley, NY), vol. 2, p. 1009.

[17] Roček, M., Shrock, R., and Tsai, S.-H. 1998 Physica A252, 505.

[18] Roček, M., Shrock, R., and Tsai, S.-H. 1998 Physica A259, 367.

[19] Shrock, R. and Tsai, S.-H. 1998 Physica A259, 315.

[20] Shrock, R. and Tsai S.-H. 1998 Phys. Rev. E58, 4332, cond-mat/9808057.

[21] Shrock, R. and Tsai, S.-H. 1999 J. Phys. A Lett. 32 L195.

[22] Shrock, R. and Tsai, S.-H. 1999 J. Phys. A 325053.

[23] Shrock, R. 1999, in the Proceedings of the British Combinatorial Conference (July, 1999); Shrock, R. 2000 Physica A, in press.

[24] H. Klüpfel and R. Shrock, unpublished; H. Klüpfel, Stony Brook thesis, July, 1999.

[25] Shrock, R. 1999, in the Proceedings of Statphys-Taiwan-99 (August, 1999).

[26] Biggs, N. L. and Shrock, R. 1999 J. Phys. A (Letts.) 32, L489.

[27] Biggs, N. L. LSE report LSE-CDAM-99-03 (May, 1999). 
[28] Biggs, N. L. LSE reports LSE-CDAM-99-05,06 (July, 1999).

[29] Baxter, R. J. 1987 J. Phys. A 20, 5241.

[30] Sands, D. A., Ph.D. Thesis, Univ. of London, 1972 (unpublished). RS thanks Prof. N. L. Biggs for kindly sending him a copy of this thesis.

[31] Shrock, R. and Tsai, S.-H. 1997 Phys. Rev. E56, 3935.

[32] Shrock, R. and Tsai, S.-H. 1998 J. Phys. A 31, 9641.

[33] Shrock, R. and Tsai, S.-H. 1999 Physica A265, 186.

[34] Sokal, A. Combin. Prob. Comput., in press.

[35] Shrock, R. and Chang, S.-C. YITP-SB-00-12.

[36] Matveev, V. and Shrock, R. 1995 J. Phys. A 285235.

[37] Shrock, R. and Tsai, S.-H. 1997 Phys. Rev. E55, 6791, Phys. Rev. E56, 4111.

[38] Wannier, G. H. 1950 Phys. Rev. 79, 357.

[39] Stephenson, J. 1964 J. Math. Phys. 5, 1009.

[40] Chang, S.-C. and Shrock, R., YITP-SB-99-58

[41] Salas, J. and Sokal, A., work in progress (private communication).

[42] Chang, S.-C. and Shrock, R. YITP-SB-00-11.

[43] Jackson, B. 1993 Combin. Prob. Comput. 2325.

[44] Thomassen, C. 1997 Combin. Prob. Comput. 6497.

[45] Brown, J. 1998 J. Combin. Theory B72, 251.

[46] Shrock, R. and Tsai, S.-H. 1997 Phys. Rev. E56, 1342. 